Received: 9 January 2018

Accepted: 23 April 2018

Published online: 03 May 2018

ENTIFIC REP

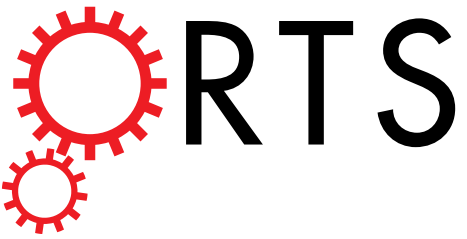

OPEN

\title{
Active Site Gate Dynamics Modulate the Catalytic Activity of the Ubiquitination Enzyme E2-25K
}

Manoj K. Rout ${ }^{1}$, Brian L. Lee ${ }^{1}$, Aiyang Lin ${ }^{2}$, Wei Xiao $\mathbb{D}^{2}$ \& Leo Spyracopoulos ${ }^{1}$

The ubiquitin proteasome system (UPS) signals for degradation of proteins through attachment of K48-linked polyubiquitin chains, or alterations in protein-protein recognition through attachment of $\mathrm{K} 63$-linked chains. Target proteins are ubiquitinated in three sequential chemical steps by a threecomponent enzyme system. Ubiquitination, or E2 enzymes, catalyze the central step by facilitating reaction of a target protein lysine with the $C$-terminus of $U b$ that is attached to the active site cysteine of the E2 through a thioester bond. E2 reactivity is modulated by dynamics of an active site gate, whose central residue packs against the active site cysteine in a closed conformation. Interestingly, for the E2 Ubc13, which specifically catalyzes $\mathrm{K} 63$-linked ubiquitination, the central gate residue adopts an open conformation. We set out to determine if active site gate dynamics play a role in catalysis for E2-25K, which adopts the canonical, closed gate conformation, and which selectively synthesizes K48-linked ubiquitin chains. Gate dynamics were characterized using mutagenesis of key residues, combined with enzyme kinetics measurements, and main chain NMR relaxation. The experimental data were interpreted with all atom MD simulations. The data indicate that active site gate opening and closing rates for E2-25K are precisely balanced.

Neurodegenerative diseases such as Alzheimer's (AD), Parkinson's (PD), Huntington's (HD) disease and amyotrophic lateral sclerosis (ALS) share a common mechanism of pathogenesis: the build-up of misfolded toxic proteins and protein aggregates in brain cells. This is due in part to impaired clearance mechanisms, whose efficiency decreases with age ${ }^{1}$. A growing body of evidence points at protein quality control by the ubiquitin proteasome system (UPS), which tags proteins with ubiquitin (Ub) for removal from the cell, as an important player in many neurodegenerative disorders ${ }^{2,3}$. The UPS system attaches lysine 48-linked (K48) polyubiquitin (polyUb) chains on target proteins, which destines them for removal from cells ${ }^{4}$. Cell cycle control ${ }^{5}$, trafficking of cell surface receptors ${ }^{6}$, and activation of transcription by NF- $\kappa B^{7}$, are regulated by attachment of K48-polyUb chains to target proteins. This post-translational modification signals for degradation of the protein by the proteasome ${ }^{8}$. Alternatively, covalent attachment of K63-polyUb chains to proteins initiates signaling cascades that lead to DNA repair $^{9-11}$, altered gene expression ${ }^{12}$, or autophagy ${ }^{13}$.

Mutations within the enzymes and proteins of the UPS contribute to the pathogenesis of disease states, including neurodegeneration, cancer, and viral infection ${ }^{14-17}$. The UPS attaches Ub to target proteins in three steps, starting with attachment to a Ub-activating enzyme, or (E1), a transfer to a Ub-conjugating enzyme (E2), with final attachment of Ub to a target by a Ub-ligase (E3) ${ }^{14}$. The UPS regulates biochemical processes using one E1 enzyme, $40 \mathrm{E} 2 \mathrm{~s}$, and hundreds of E3s, which provide recognition of targets ${ }^{18}$. The Ub signal has a diverse architecture, variants include attachment of single, or multiple Ub molecules to different sites on a protein, or polyubiquitination through single (homotypic), or variable types (heterotypic) of Ub-Ub peptide bonds ${ }^{19}$. Seven types of homotypic polyUb chains are formed through seven surface lysines on $\mathrm{Ub}^{19,20}$; the most highly studied chains are those linked through K48 or K63. An understanding of the remaining chains is less well developed, though generally, it is likely that different chains are involved in different physiological processes. The topological variety for the Ub signal likely gives organisms a biological advantage for achieving specificity in different signaling cascades and biological functions.

${ }^{1}$ Department of Biochemistry, Faculty of Medicine and Dentistry, University of Alberta, Edmonton, Alberta, T6G 2H7, Canada. 'Department of Microbiology and Immunology, University of Saskatchewan, Saskatoon, Saskatchewan, S7N 5E5, Canada. Correspondence and requests for materials should be addressed to L.S. (email: leo.spyracopoulos@ualberta.ca) 


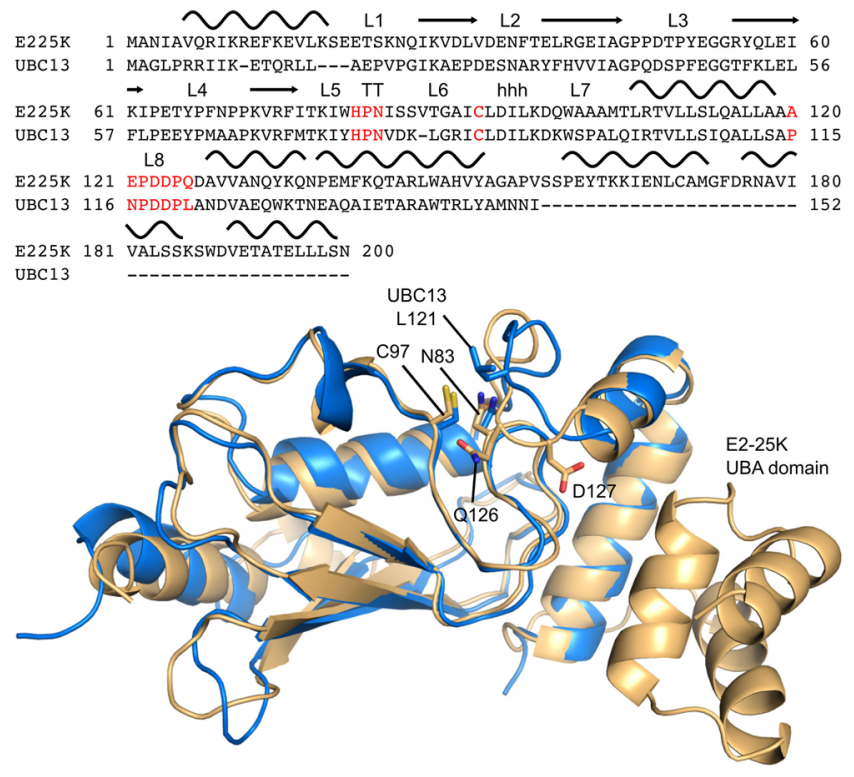

Figure 1. Sequence (top) and structure (bottom) alignments for E2-25K and Ubc13. Secondary structures and loop numbers are shown for the sequence alignment, with the HPN motif, catalytic cysteine, and the active site DPNPxxPL loop motif highlighted in red. The structures of E2-25K (gold) and Ubc13 (blue) correspond to PDB IDs $3 \mathrm{~K} 9 \mathrm{O}$ and 1J7D, respectively, key residues and the E2-25K UBA domain are indicated.

The E2 enzyme E2-25K is notable for its potential involvement in $\mathrm{AD}^{21}$ and $\mathrm{HD}^{22,23}$. Paradoxically, E2-25K catalytic activity has been shown to increase cell death in AD and HD models, potentially through proteasome inhibition $^{21}$. In HD models, misfolded mutant huntingtin (mHTT) protein is tagged with K48-polyUb and removed by the UPS, or alternatively, tagged with K63 polyUb chains for degradation through the autophagic pathway ${ }^{24}$. Disruption of these processes, or an imbalance between K48/K63 ubiquitination can lead to accumulation of toxic $\mathrm{mHTT}^{25}$. In this regard, inhibition of E2-25K has been shown to decrease the build-up of toxic mHTT fragments $^{23}$. The mechanism is currently unknown but may be a result of the formation of unanchored polyUb chains that bear $\mathrm{UBB}+$, a frameshift Ub mutant that is known to be a potent inhibitor of the proteasome ${ }^{21}$.

Generally, these observations indicate that E2 enzymes possess precise reaction rates that are critical for maintaining cellular homeostasis. E2 enzymes catalyze the central step in the UPS, and achieve a $10^{6}$-fold acceleration for the second step of the UPS, or the aminolysis of the Ub thioester bond (nucleophilic attack of lysine on the E2 Ub thioester bond) ${ }^{17,26}$. E2s orchestrate many functions, including shifting from initiation of polyUb chain synthesis to chain elongation, selection of Ub lysines for chain links, and precise timing of chain release to control chain length ${ }^{27}$. Ultimately, E2-dependent ubiquitination of specific proteins is regulated by weak binding of substrates and E3 Ub ligases ${ }^{16,28-30}$. Enzyme kinetic studies have revealed that specificity for E2 enzymes is achieved through variability in chemical mechanism ${ }^{16,17,26}$. Modest differences in the K48-polyUb chain building rate by the SCF Ub ligases gives rise to a large difference in the ability to signal for protein degradation ${ }^{16}$. Additionally, modest decreases in the rate of K63-polyUb chain synthesis by the K63-specific E2 Ubc13 can give rise to defects in the ability of cells to respond to DNA damage ${ }^{26}$. From a mechanistic perspective, the reaction rate of Ubc13 is regulated by the closing and opening dynamics of an active site gate, which connects the $\alpha 2$ and $\alpha 3$ helices, and buttresses the active site cysteine ${ }^{26}$. The gate fluctuations between the open and closed states are precisely balanced; small deviations in gating impair the ability of cells to respond to DNA damage $\mathrm{e}^{17,26}$.

Interestingly, the active site loop from free $\mathrm{Ubc13}$ adopts a conformation that is not typically observed for E2

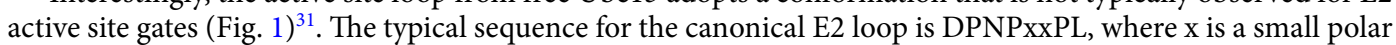
or charged residue ${ }^{31}$. Two conformations have been observed for the active site loop, with the leucine residue exposed in $\mathrm{Ubc}^{32} 3^{32}$, but packed against the core fold in canonical E2s, maintaining the loop in a "closed", catalytically active conformation ${ }^{30}$. Ultimately, Ubc13 adopts a closed conformation to facilitate catalysis, as observed for the structure of the Mms2-Ubc13 holoenzyme with covalently attached "donor" Ub ${ }^{33,34}$. Mutation of the loop leucine in Ubc13 (L121) to a series of hydrophobic residues of increasing size (G, A, V, I), revealed that it functions to promote solvation substitution at the active $s^{2} \mathrm{e}^{35}$, in order to enhance electrostatic stabilization of the transition state $^{26}$. Furthermore, mutation of the key hinge residue (A122) within the loop to glycine, revealed the key role of dynamics in the catalytic activity of Ubc13 ${ }^{26}$.

The active site loop in E2-25K adopts the canonical closed conformation ${ }^{36}$, however, the typical leucine residue within the loop is glutamine (Fig. 1). To understand the role of active site gate residues in E2-25K catalysis, we mutated the central loop glutamine to leucine, isoleucine, alanine, valine, and glycine, and conducted enzyme kinetics assays. To assess the role of loop dynamics on enzyme activity, we mutated the hinge residue D127 to glycine; this is the equivalent residue to A122 in Ubc13. In addition to enzyme kinetics measurements, the impact of mutations on the structural and dynamics properties of E2-25K were studied using NMR backbone resonance chemical shift changes and ${ }^{15} \mathrm{~N}$ NMR relaxation. Atomic views of the loop motions and estimates for the barrier 


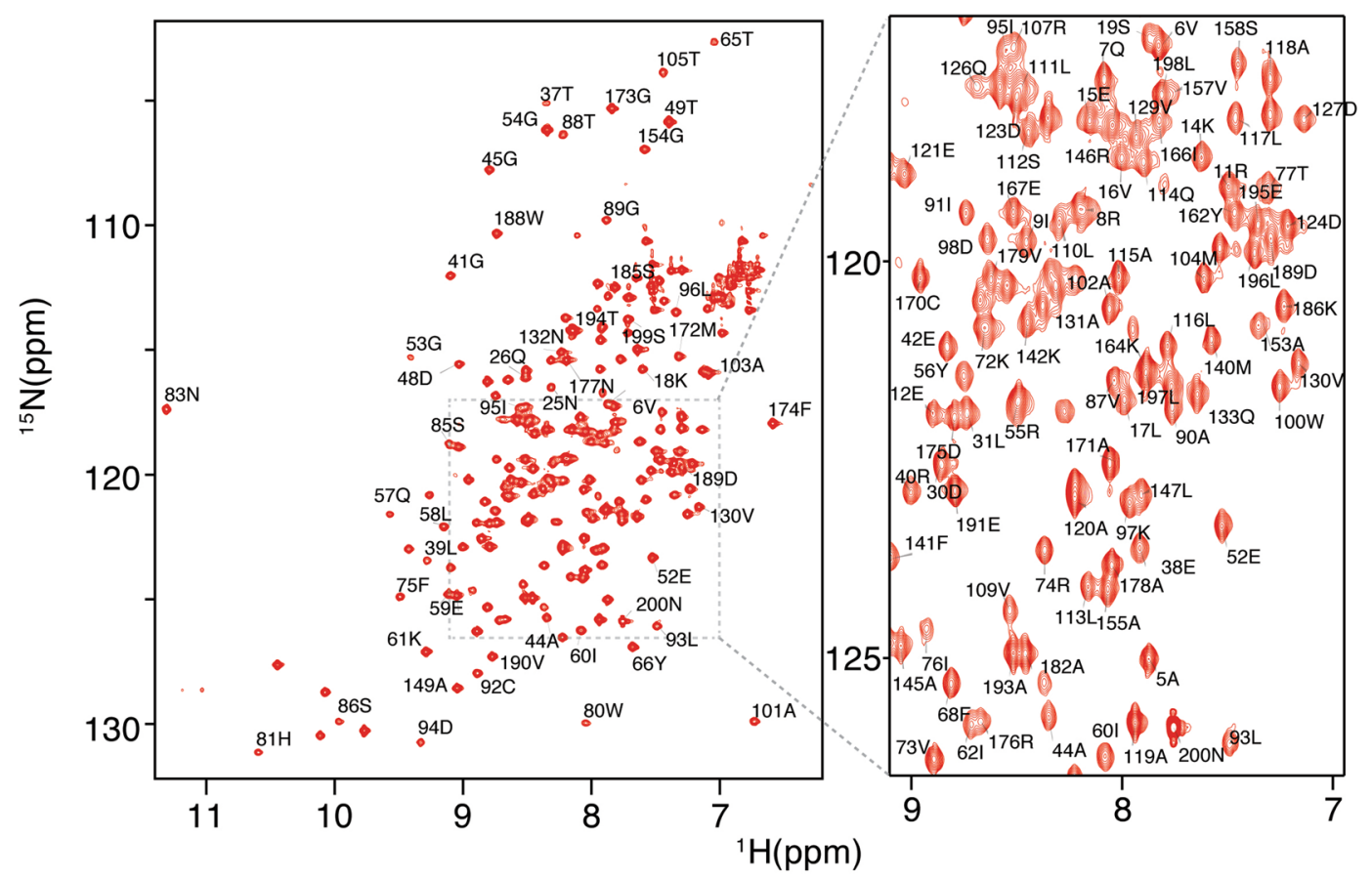

Figure 2. ${ }^{15} \mathrm{~N}-{ }^{1} \mathrm{H}^{\mathrm{N}} \mathrm{HSQC}$ spectrum for E2-25K.

heights of gate opening for E2-25K as well as the Q126L and D127G mutants, were obtained from all atom molecular dynamics simulations. This combined approach has revealed key mechanistic and dynamic differences between canonical and non-canonical active site loops in E2 enzymes.

\section{Results}

NMR derived secondary structures for active site E2-25K gate mutants are similar to wildtype. The ${ }^{1} \mathrm{H}^{-15} \mathrm{~N}$ HSQC NMR spectrum for wildtype E2-25K is shown in Fig. 2. Chemical shift perturbations $(\Delta \delta)$ indicate that the majority of residues are unaffected by the Q126L and D127G substitutions, apart from residues immediately adjacent to the active site Cys (residues 75-95), and the active site loop (residues 120-135), (Fig. 3). For the D127G mutant, the chemical shift perturbations within the active site loop arise from changes in local environment resulting from the mutations, whereas the larger $\Delta \delta$ values in the active site (residues 75-95) reflect the close coupling of the active site to the buttressing active site loop (residues 120-135). For Q126L, there are fewer changes in chemical shift for the loop region, and changes are less dispersed across the loop in comparison to D127G. The secondary structures of E2-25K and the Q126L/D127G mutants were determined from main chain ${ }^{15} \mathrm{~N},{ }^{1} \mathrm{H}^{\mathrm{N}}, \mathrm{CO}$ and $\mathrm{C}_{\alpha}$ chemical shifts using the CSI 3.0 program (Supplementary Fig. S1). The secondary structures for Q126L and D127G are similar to those for wild type, indicating that the structural and dynamic changes for these mutants are localized to the active site and gating loop.

NMR monitored titration indicates that the interaction of Ub with E2-25K occurs with weak affinity and fast kinetics. The UBA domain from E2-25K, in conjunction with the core UBC domain, functions to position an "acceptor" Ub to facilitate catalysis of the synthesis of K48-linked Ub chains. We monitored the interaction of Ub with E2-25K using ${ }^{1} \mathrm{H}^{\mathrm{N}}-15 \mathrm{~N}$ HSQC NMR spectroscopy (Fig. 4a). The largest chemical shift perturbations $(\Delta \delta)$ include residues G173, F174, and V190 within the UBA domain (Fig. 4b), accompanied by significant $\Delta \delta$ values that exceed the average chemical shift by $1 \sigma$ (Fig. $4 \mathrm{c}$ ). From the titration, $\Delta \delta$ values yield an average dissociation constant $\left(K_{D}\right)$ of $206 \pm 30 \mu \mathrm{M}$ for 1:1 protein-protein binding (Fig. $\left.4 \mathrm{~d}\right)$. It has previously been shown that binding of Ub by the human E2-25K UBA domain alone is extremely weak, $K_{D} \sim 1.2 \pm 0.3 \mathrm{mM}^{37}$. The $K_{D}$ value we measure for Ub binding to intact human E2-25K is consistent with the $K_{D}$ of $228 \pm 60 \mu \mathrm{M}$ for Ub binding to yeast E2-25K, measured using NMR methods $s^{36,38}$, and the apparent $K_{D}$ (or $K_{M}$ value) for Ub binding of $\sim 350 \mu \mathrm{M}$ derived from $\mathrm{K} 48-\mathrm{Ub}_{2}$ chain synthesis assays ${ }^{39}$. These dissociation constants for yeast and human E2-25K indicate that both the UBC and UBA domains interact with ubiquitin, demonstrating the fundamental principle of catalysis by proximity and orientation for E2-25K. Using spectra from our NMR titration, we also conducted ${ }^{15} \mathrm{~N}$ NMR lineshape analysis for the main chain amide of G173, to determine rate constants for the interaction with $k_{o n}=2.9 \times 10^{7} \mathrm{M}^{-1} \mathrm{~s}^{-1}$ and $k_{\text {off }}=5886 \mathrm{~s}^{-1}$ (Fig. 4e). These values were subsequently used in the rate laws describing the kinetics of enzyme assays for synthesis of $\mathrm{K}_{48}-\mathrm{Ub}_{2}$ chains by $\mathrm{E} 2-25 \mathrm{~K}$, described in more detail below, and within the Methods.

Conjugation of Ub to E2-25K Q126L by E1 enzyme is substantially enhanced compared to wildtype. The first step of ubiquitination cascade is the E1-catalyzed, ATP-dependent covalent attachment of the active site cysteine of an E2 with the C-terminal carboxyl of Ub. Under conditions where the concentrations of E2 are the same, accounting for E1 concentrations gives apparent rate constants $\left(k_{a p p}=k_{\mathrm{obs}} /[\mathrm{E} 1]\right)$ for conjugation of Ub 

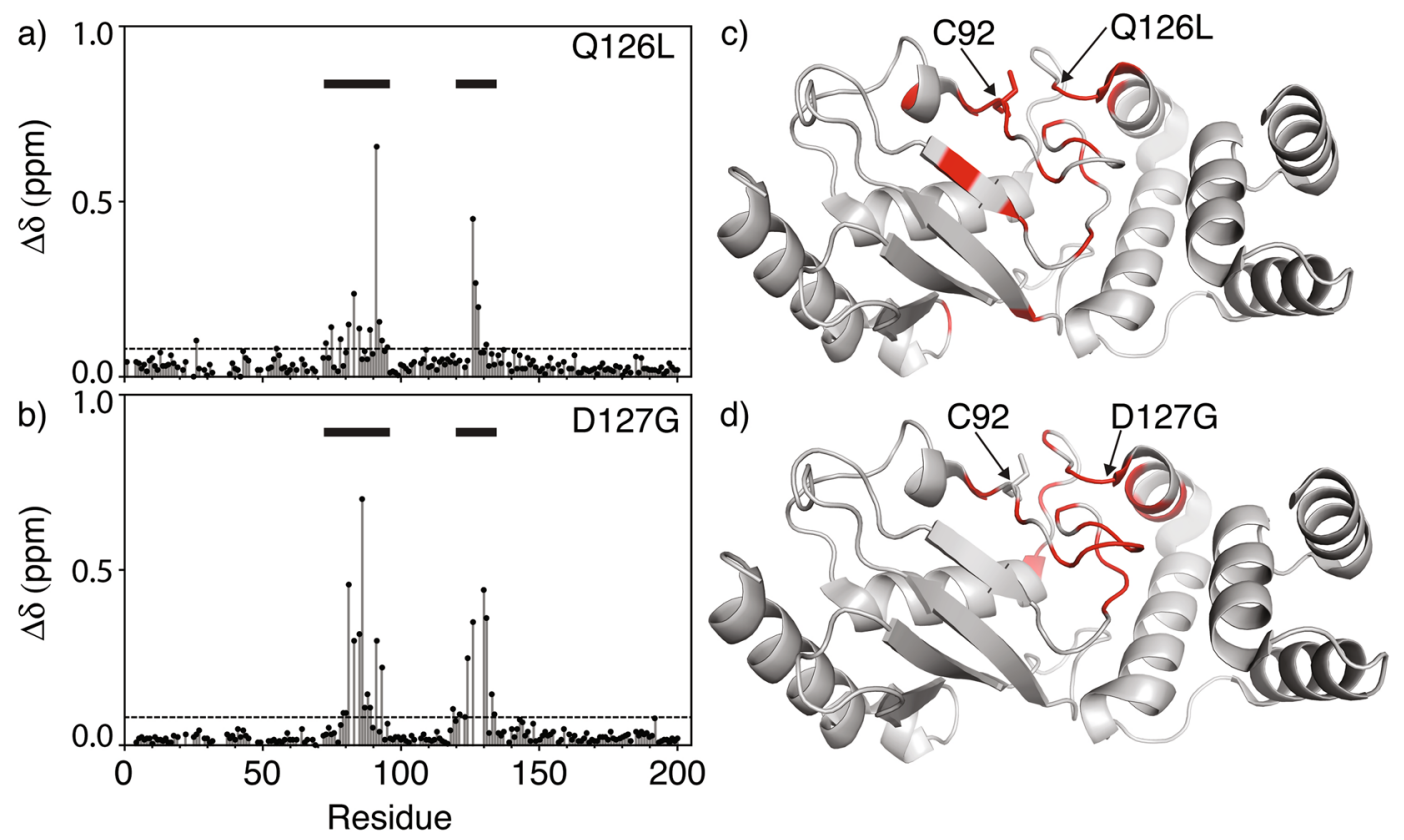

Figure 3. ${ }^{1} \mathrm{H}^{\mathrm{N}}-{ }^{15} \mathrm{~N}$ chemical shift perturbations $(\Delta \delta)$ for E2-25K Q126L and D127G active site loop mutations. The active site, residues 75-95, and the active site loop, residues 120-135, are indicated with horizontal bars. Residues with significant chemical shift changes $(>\Delta \delta+1 \sigma)$ are shown on the main chain structure (red) for Q126L (c) and D127G (d).

to $\mathrm{E} 2-25 \mathrm{~K}$ are $0.041 \pm 0.002,0.0075 \pm 0.0003$, and $1.8 \pm 0.3\left(\times 10^{6} \mathrm{M}^{-1} \mathrm{~min}^{-1}\right)$ for wildtype, D127G, and Q126L (Figs. 5a,b and S2). Interestingly, the value of $k_{a p p}$ for Q126L comparable to the previously measured value of $1.4 \pm 0.1\left(\times 10^{6} \mathrm{M}^{-1} \mathrm{~min}^{-1}\right)$ for wildtype $\mathrm{Ubc}^{2} 3^{26}$. The competing thioester hydrolysis reaction is substantially slower and does not impede aminolysis, with hydrolysis rates of $\sim 0.001 \mathrm{~min}^{-1}$, as previously observed for $\mathrm{Ubc}^{2} 3^{26}$.

Aminolysis of E2-25K Q126 thioester is substantially enhanced compared to wildtype. The aminolysis of $\mathrm{E} 2 \sim \mathrm{Ub}$ thioester by the nucleophile L-lysine provides a straightforward reaction to facilitate comparison of active site gate mutants ${ }^{26,40}$ (Fig. 6a). The observed catalytic rates of aminolysis $\left(k_{\text {obs }}\right)$ using $30 \mathrm{mM}$ lysine were $0.19 \pm 0.03,0.05 \pm 0.01$, and $1.3 \pm 0.1 \mathrm{~min}^{-1}$ for wildtype $\mathrm{E} 2-25 \mathrm{~K}$, and the D127G and Q126L mutants, respectively (Fig. 6b). To understand the role of Q126 in solvation substitution at the active site, we mutated Q126 to the series A, V, I, L, and measured the dependence of aminolysis on lysine concentration. This facilitated determination of the second order rate constant $k_{\text {cat } 2}$, which ranges from $0.05-1\left(\times 10^{8} \mathrm{M}^{-2} \mathrm{~s}^{-1}\right)$ (Fig. $\left.6 \mathrm{c}\right)$. There is an approximately linear increase in $k_{\text {cat2 }}$ for the mutants Q126A, Q126V, and Q126I with respect to average accessible surface area (ASA) of the side chain (Fig. 6d). Whilst the ASA value of leucine is only marginally larger compared to isoleucine, the $k_{\text {cat } 2}$ value is substantially larger. This result is consistent with kinetic studies using the yeast homolog of E2-25K, Ubc1, for which the mutant Q122L has an increased rate for ubiquitination of sea urchin cyclin B substrate, in comparison to wild type $\mathrm{Ubc} 1^{39}$. The $k_{\text {cat } 2}$ value for lysine aminolysis catalyzed by wildtype E2-25K is fourfold greater than that previously measured for $\mathrm{Ubc} 13^{26}$, suggesting mechanistic differences between different E2 enzymes.

E2-25K Q126L and D127G mutants show impaired rates of K48-Ub $\mathrm{H}_{2}$ synthesis. Binding of Ub by the UBA and UBC domains from E2-25K facilitates catalysis of the synthesis of K48-linked Ub chains in the absence of an E3 Ub ligase ${ }^{41}$. A schematic representation of the kinetic scheme for chain synthesis, which includes concomitant hydrolysis of the thioester bond, is shown in Fig. 7a. To understand the mechanistic aspects of the active site gate for $\mathrm{E} 2-25 \mathrm{~K}$, we measured the kinetics of $\mathrm{K} 48-\mathrm{Ub}_{2}$ chain formation (Figs. $7 \mathrm{~b}$ and S2). The rate constant for $\mathrm{K} 48-\mathrm{Ub}_{2}$ synthesis catalyzed by wildtype $\mathrm{E} 2-25 \mathrm{~K}, k_{\mathrm{Ub} 2}$, is $0.002 \mathrm{~s}^{-1}$, comparable to the value of $0.007 \mathrm{~s}^{-1}$ for catalysis of $\mathrm{K} 63-\mathrm{Ub}_{2}$ synthesis by the $\mathrm{Mms} 2 / \mathrm{Ubc} 13$ protein complex ${ }^{17}$. Interestingly, $k_{\mathrm{Ub} 2}$ for wildtype is about fourfold greater than for Q126L (Fig. 7c). The gate mutants Q126I, Q126V, and Q126A are partially impaired with respect $\mathrm{K} 48-\mathrm{Ub}_{2}$ chain formation (Fig. 7c). Similar to the results for aminolysis by lysine, the rate of $\mathrm{K} 48-\mathrm{Ub}_{2}$ chain synthesis follows a roughly linear increase as a function of ASA values for the hydrophobic side chains (Fig. 7c). Whilst catalysis of aminolysis is only partially impaired for the E2-25K D127G mutant, K48-Ub synthesis is completely impaired.

NMR relaxation measurements indicate increased loop flexibility for E2-25K D127G. ${ }^{15} \mathrm{~N}-R_{1},-R_{2}$, and NOE NMR relaxation rates were measured at $600 \mathrm{MHz}$ to assess the main chain dynamics for E2-25K and the active site mutants on the pico- to millisecond timescale (Supplementary Fig. S3). The overall rotational correlation times for wildtype, Q126L, and D127G E2-25K were determined from the $R_{2} / R_{1}$ ratio and range from $16-18 \mathrm{~ns}$. In 
a)

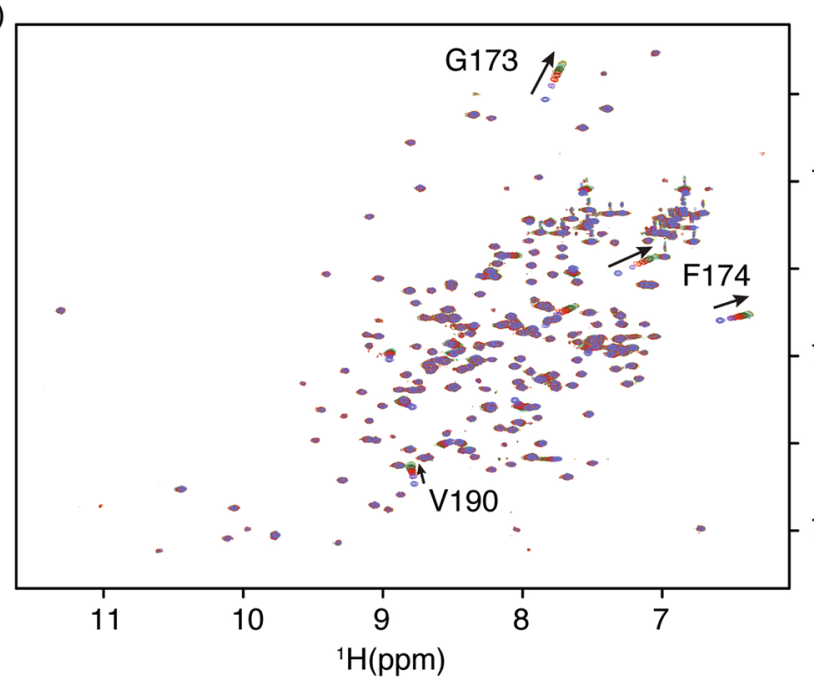

b)

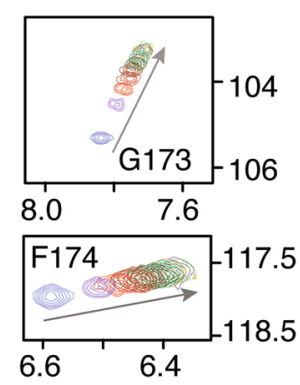

130

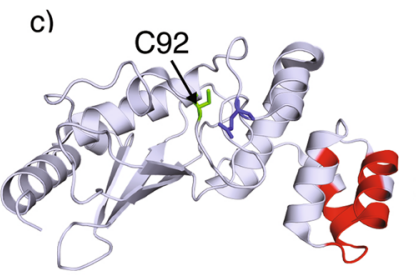

UBA d)

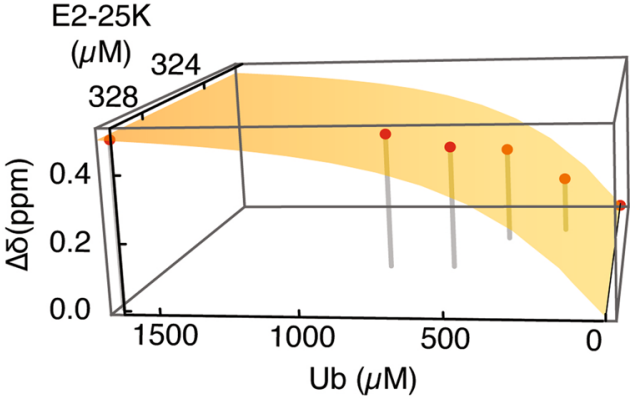

e)

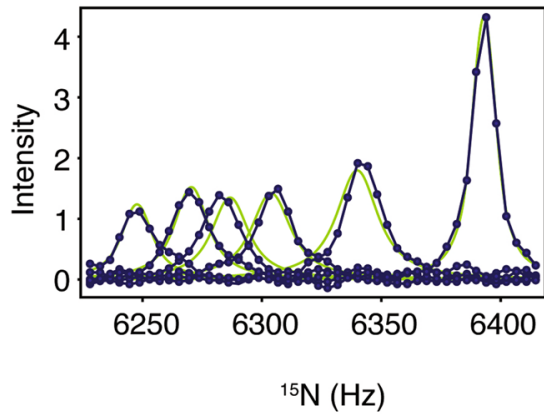

Figure 4. (a) Superposition of E2-25K ${ }^{1} \mathrm{H}-{ }^{15} \mathrm{~N}$ HSQC NMR spectra upon titration with Ub, arrows indicate chemical shift changes with increasing Ub concentration. (b) Expansion of the spectra around G173/F174, residues experiencing significant chemical shift changes upon interaction with Ub, arrows indicate increasing Ub concentration. (c) Significant chemical shift perturbations $(>\Delta \delta+1 \sigma$, red) upon Ub binding, mapped onto the main chain structure of E2-25K. The active site C92 (green) and gate residue Q126 (blue) are shown in the stick representation. (d) Chemical shift changes for G173 upon titration with Ub fit to a 1:1 binding isotherm, experimental shifts are shown as points, and the best fit as a surface. (e) ${ }^{15} \mathrm{~N}$ lineshape analysis for residue G173 with experimental data shown as points (blue), and the best fits shown as green lines.

a)

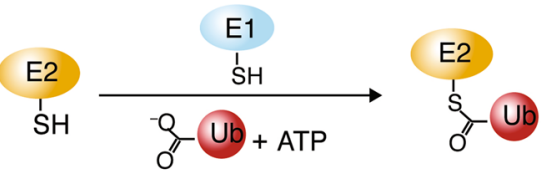

b)

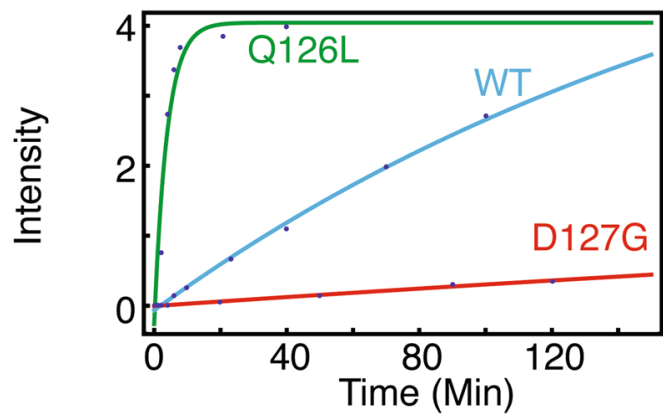

Figure 5. Schematic presentation of ATP-dependent E2-25K thioester formation catalyzed by E1 enzyme (a). Fluorescence intensities for thioester synthesis are shown as blue dots, with best fits shown as lines (b).

contrast to our previous studies of the active site loop for the E2 $\mathrm{Ubc} 13^{26}$, resonance overlap and low signal to noise ratios for a number of peaks in the ${ }^{1} \mathrm{H}-{ }^{15} \mathrm{~N}$ HSQC NMR spectra hinders comprehensive analysis of loop relaxation rates. However, for the gate mutant $\mathrm{D} 127 \mathrm{G},{ }^{15} \mathrm{~N}-R_{1},-R_{2}$, and $\mathrm{NOE}$ values at residue 127 indicate increased flexibility in comparison to wildtype (Supplementary Figs. S3 and S4). For the gate mutant Q126L, resonance overlap prevents 
a)

c)

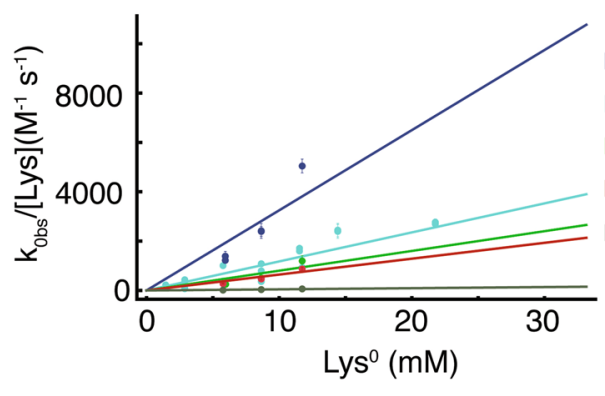

b) WT $\square 127 G \quad$ Q126L $\square$ Q126G

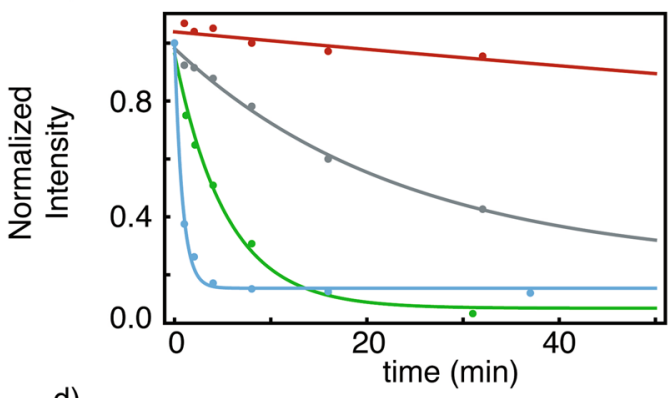

d)

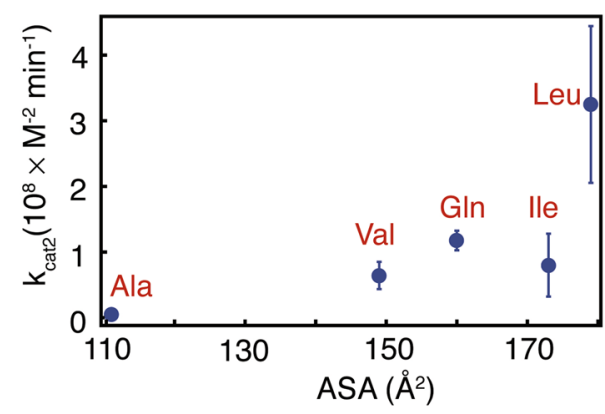

Figure 6. Aminolysis of E2-25K thioester by lysine. For the general reaction (a), the kinetics of thioester aminolysis by $30 \mathrm{mM}$ lysine are shown in (b), where apparent rates are derived from exponential fits of the data (lines). Second order rate constants $\left(k_{\text {cat } 2}\right)$ derived from the concentration dependence of aminolysis kinetics by neutral lysine are shown in (c), and plotted as a function of side chain accessible surface area for position 126 of wildtype E2-25K wildtype (Q), and Q126L, Q126I, Q126V, and Q126A mutants.

a)
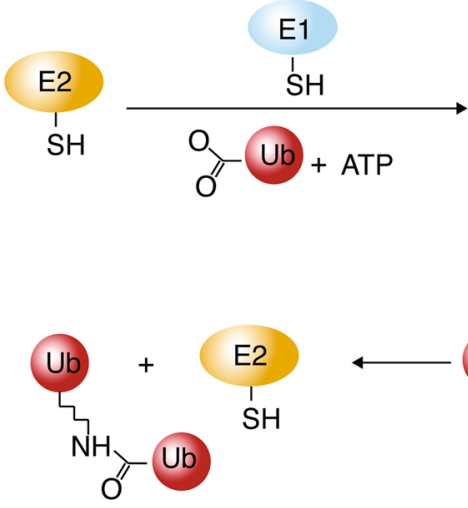

b)

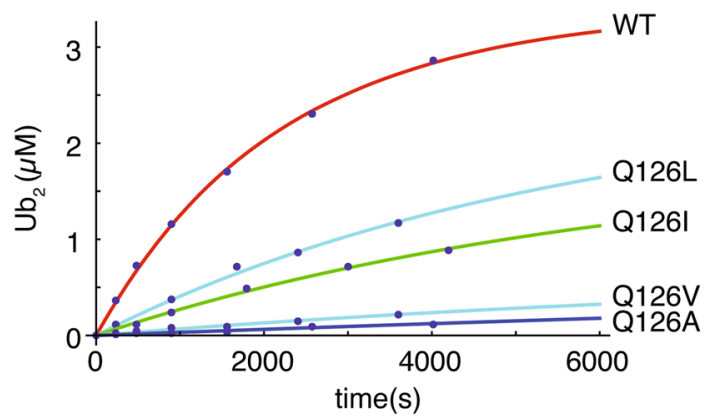

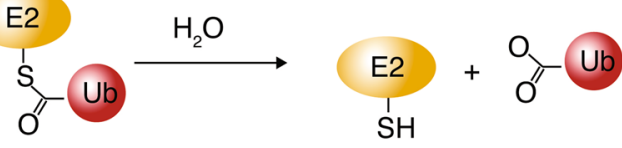

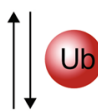

^Ub

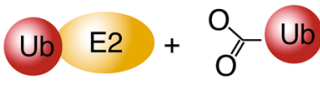

Figure 7. (a) Reaction scheme for catalysis of K48-linked $\mathrm{Ub}_{2}$ synthesis by E2-25K (a). (b) Kinetics of K48linked $\mathrm{Ub}_{2}$ chain formation catalyzed by $\mathrm{E} 2-25 \mathrm{~K}$ and active site gate mutants, with associated fits to yield the $k_{\mathrm{Ub} 2}$ rate constants, plotted as a function of side chain ASA values for gate mutants (c). 


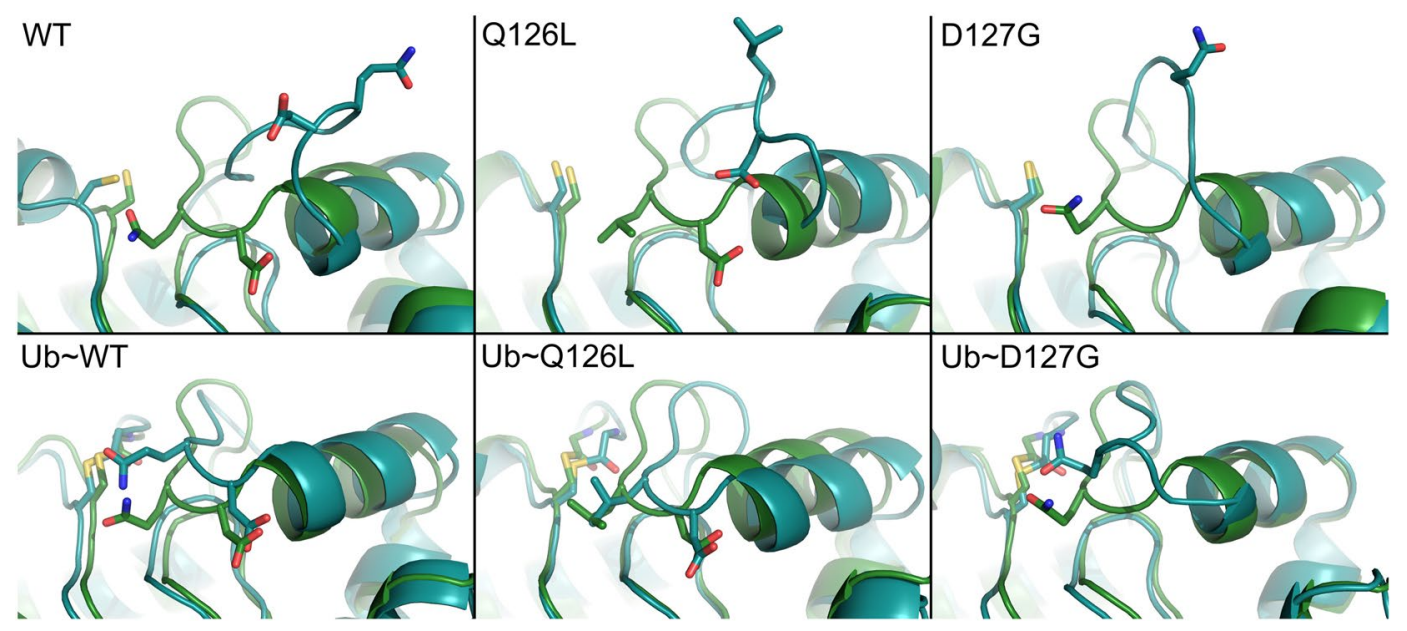

Figure 8. Stochastic gating for the active site loop of E2-25K and various mutants, in the free and Ubthioester conjugated states. The starting conformations and representative open gate conformations are shown. Gate opening was observed in the free enzyme (top row), and the ubiqutin thioester-linked wildtype and D127G enzymes (bottom row). C92, Q/L126, D127 and G76 from thioester linked Ub are shown in the stick representation.

comprehensive comparison to wildtype, however, ${ }^{15} \mathrm{~N}-R_{1}, R_{2}$, and NOE values at residues 123 and 126 are not indicative of increased flexibility for the loop.

MD simulations indicate that the barrier to gate opening for E2-25K D127G decreases compared to wildtype. The dynamics for E2-25K and the active site gate mutants Q126L and D127G were probed using MD simulations. The active site gate is generally observed to undergo stochastic fluctuations between two states, a closed state for which the distance between the $\mathrm{C}_{\alpha}$ atom of $\mathrm{C} 92$ within the active site and the $\mathrm{C}_{\alpha}$ atom of Q126 at the center of the active site gate is $\sim 6-8 \AA$, and an open state where the distance increases to $\sim 12-16 \AA$ (Fig. 8). The rates of gate opening are slow over the course of the $400-800$ ns simulation times, with only a few transitions to the open state observed for the various proteins; two representative MD runs for each of wildtype, D127G, and Q126L are shown in Fig. 9. The total number of transitions over the total simulation times can be used to provide estimates for the barrier heights of gate opening of $9.2,8.0$, and $7.9 \mathrm{kcal} / \mathrm{mol}$ for wildtype, Q126L, and D127G, respectively, with similar barrier heights for closing. We also determined the barrier heights for opening using steered MD simulations, which gave underestimated values in comparison to counting transitions from the MD runs, but a similar decreasing trend for the barrier to gate opening with values of 6.1,5.6, and $3.8 \mathrm{kcal} / \mathrm{mol}$ for wildtype, Q126L, and D127G, respectively (Fig. 10). For simulations involving wildtype, and D127G E2-25K Ub with the C-terminus of Ub covalently attached to the active site Cys through a thioester bond, and Ub in the "closed" conformation, the rates of gate opening are also slow over the 400-800 ns simulation times, with only a few opening transitions observed, similar to the non-thioester simulations. The total number of open and closed transitions over the course of multiple $400-800 \mathrm{~ns}$ simulations gave barrier heights for opening of 7.0 and $7.5 \mathrm{kcal} / \mathrm{mol}$ for D127G and wildtype, respectively. The magnitude of opening is $\sim 2-3 \AA$ smaller than the non-thioester enzyme but remains sufficient to allow product release. We did not observe opening of the gate for Q126L Ub in MD simulations. However, given that the experimental rates of catalysis for this mutant are faster than wildtype, release of the $\mathrm{C}$-terminus of $\mathrm{Ub}$ from the active site is not rate limiting. In general, the hydrogen bond between the carbonyl oxygen of the E2-25K Ub thioester bond and the side chain amide proton of N83 remained stable throughout the simulations for wildtype, Q126L, and D127G E2-25K. Interestingly, during the MD simulations, H81 from the canonical HPN motif of E2 enzymes was observed to rapidly adopt the side chain conformation that stabilizes a tight turn, rather than interact with the side chain of N83, as previously observed using NMR studies ${ }^{42}$.

${ }^{15} \mathrm{~N}-R_{1},-R_{2}$, and NOE NMR relaxation values were calculated from the MD simulations for comparison to experimental values acquired at $600 \mathrm{MHz}$. Twenty nanosecond windows corresponding to either the closed or open states were taken from $\sim 800 \mathrm{~ns}$ trajectories for E2-25K and the Q126L and D127G mutants and used to determine the correlation functions for fluctuations of the main chain amide ${ }^{15} \mathrm{~N}-{ }^{1} \mathrm{H}^{\mathrm{N}}$ vectors, corresponding to either the open or closed states. The correlation functions were fit to a five-parameter exponential decay function to extract correlation times ${ }^{43}$. These correlation times were subsequently used in a five-parameter spectral density function, along with the experimentally determined correlation times $\left(\tau_{\mathrm{c}}\right)$ for overall molecular tumbling of E2-25K and the active site mutants, to calculate ${ }^{15} \mathrm{~N}-R_{1}, R_{2}$ and NOE relaxation parameters for the open and closed states, as previously described ${ }^{26,43}$. The relaxation rates for a given state were then averaged over the number of windows taken from the trajectory and weighted according to the fraction of closed and open states observed during the MD simulations to produce the final rates (Supplementary Figs. S4 and S5). The MD derived relaxation rates indicate that pico to nanosecond main chain dynamics for residues 123-127 of the gating loop from wildtype and Q126L E2-25K are similar, whereas the loop is more flexible at the hinge position 127 for the D127G mutant, evident through decreased MD-derived ${ }^{15} \mathrm{~N}-R_{1}, R_{1}$, and NOE values. Detailed comparison between MD-derived ${ }^{15} \mathrm{~N}-R_{1}, R_{1}$, and NOE values and experimental values 

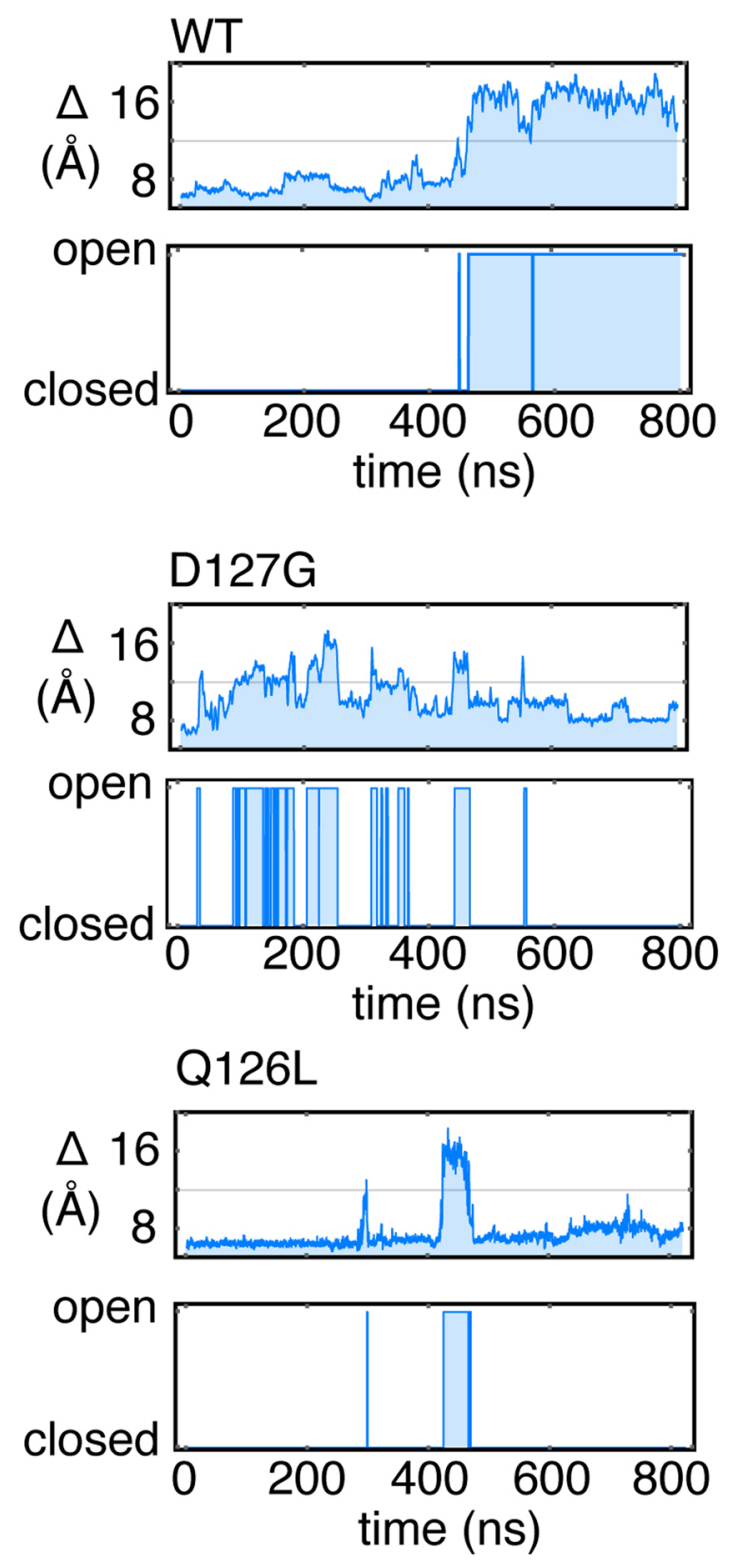

Figure 9. Stochastic gating for the active site loop of E2-25K and various mutants, in the free state derived from MD simulations. The upper panels for each protein indicate the distance between the $\mathrm{C}_{\alpha}$ of $\mathrm{C} 92$ and the $\mathrm{C}_{\alpha}$ of Q126 or Q126L, with the cut-off value between open and closed indicated by a line. The lower panels indicate digitization to a two state Markov chain.

is hampered as a result of resonance overlap and low signal to noise ratios for the NMR data. However, experimental and MD-derived ${ }^{15} \mathrm{~N}-R_{2}$ and NOE values indicate increased flexibility at hinge residue 127 for the D127G mutant (Supplementary Figs. S4 and S5).

\section{Discussion}

The selective catalysis of covalent isopeptide bonds between $\mathrm{Ub}$ and a multitude of target proteins is managed by the three-component ubiquitination enzyme cascade ${ }^{20}$. E2 enzymes catalyze the central step of ubiquitination, linking activation of the C-terminus of Ub with final attachment to target lysines with accessory E3 Ub ligases ${ }^{27,44}$. E2 enzymes possess a loop that buttresses the active site; this loop typically adopts a closed conformation, with a conserved region composed of the sequence PXXPY, where X corresponds to Asp, Asn, Ser, Ala, Glu and His ${ }^{31}$. In general, $\mathrm{Y}$ is hydrophobic (Ala or Leu), with the exception of E2-25K and its yeast homolog UBC1, for which this residue is hydrophilic (Q126). For canonical E2 enzymes, including E2-25K, the residue at position Y is generally packed against the active site Cys, in a closed conformation ${ }^{31}$. In contrast, for the E2 enzyme dedicated to catalyzing synthesis of K63-linked chains, Ubc13, L121 at position Y is typically fully exposed to solvent, in an open conformation ${ }^{32}$.

In order for a substrate lysine to be covalently attached to the C-terminus of Ub, the C-terminal tail must insert into the active site cleft of an activated E2, in other words, an E2 with the C-terminus of Ub conjugated to 


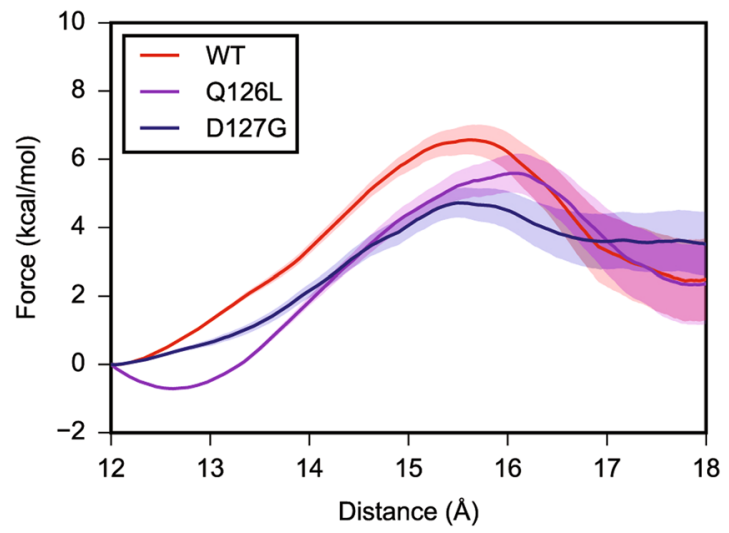

Figure 10. Steered MD simulations for opening of the active site gate of E2-25K and various mutants, in the free state. The $x$-axis indicates the distance between the $\mathrm{C}_{\alpha}$ of Q126 or Q126L, and the geometric center of the protein, not including the loop.

the active site Cys through a thioester bond. Upon entry into the active site, the carbonyl oxygen of the thioester bond forms a hydrogen bond to a buried, conserved Asn residue, and nucleophilic attack of the thioester by the deprotonated lysine amino group can occur ${ }^{30,45}$. Given the close packing between the loop and the active site, entry of the C-terminus of $\mathrm{Ub}$ into the active site cleft requires conformational rearrangement and opening of the loop, as previously observed for $\mathrm{Ubc}^{2} 3^{26}$. The opening of the active site cleft, and close packing of thioester-linked $\mathrm{Ub}$ on the underside of the E2, is facilitated by E3 Ub ligase accessory proteins, though the mechanistic basis is not clear ${ }^{29}$.

In this study, we examined the role of key active site residues in the catalytic mechanism for E2-25K in the absence of an E3 Ub ligase. Aminolysis of the E2 Ub thioester by L-lysine provides a convenient method to compare the catalytic activity of different E2s, or E2 mutants, in the absence of substrate specific effects ${ }^{17,40}$. Interestingly, mutation of the key glutamine residue at position $\mathrm{Y}$ to leucine, results in a substantial increase in the rate of thioester formation by E1, as well as the second order rate constant for aminolysis. The mechanistic importance of this position is highlighted by the roughly linear increase in the second order rate constant of aminolysis with respect to average hydrophobic accessible surface upon systematic mutation of position $\mathrm{Y}$ of the loop to A, V, I, and L, combined with the increased rate of aminolysis upon mutation to leucine. A hydrophobic residue at position $Y$ facilitates interactions that stabilize the closed conformation. Furthermore, the linear dependence of the second order rate constant for aminolysis upon systematic mutation of Q126 to increasingly hydrophobic residues indicates that position Y plays a role in solvation substitution. That is, the enhancement of electrostatic interactions within an enzyme active site through exclusion of solvent ${ }^{35}$, as we previously observed for this position in the E2 $\mathrm{Ubc} 13^{17}$. That these effects are largely static in nature is corroborated by our combined MD and NMR relaxation studies, which indicate that there are no substantive changes in flexibility for the Q126L mutant in comparison to wildtype. It is noteworthy that the Q126L mutant is impaired with respect to catalysis of the synthesis of $\mathrm{K} 48-\mathrm{Ub}_{2}$ chains. This observation indicates that $\mathrm{E} 2-25 \mathrm{~K}$ is tailored to synthesize K48-linked Ub chains, with Q126 participating in specific interactions with an acceptor Ub bearing the nucleophilic K48 which ultimately reacts with the donor $\mathrm{Ub} \sim \mathrm{E} 2-25 \mathrm{~K}$ thioester bond. Our findings are consistent with kinetic and mutational studies for Ubc1, the yeast homolog of E2-25K, for which position Y is Q122, and is critical for synthesis of K48-linked Ub chains, but which shows significantly enhanced ubiquitination of cyclin B for the Q122L mutant ${ }^{39}$. Thus, position $\mathrm{Y}$ is unique amongst active site residues, as it enhances, rather than diminishes catalysis of aminolysis. For example, the active site residues N83 and D124 in E2-25K are conserved in E2s. N83 directly stabilizes the oxyanion, and D124 stabilizes the developing charge on the nucleophilic substrate lysine for the zwitterionic tetrahedral transition state ${ }^{17,30,46}$. Various mutations of these key active site residues reduce catalytic activity ${ }^{30,46,47}$. D123 in E2-25K is expected to position substrate through ionic interactions with R74 from the C-terminus of the donor $\mathrm{Ub}$, as observed for $\mathrm{UbcH} 5 \mathrm{~A}$, with mutations that disrupt this interaction reducing catalytic activity ${ }^{30}$.

In addition to electrostatic effects discussed above, reactions with the nucleophile L-lysine facilitate studies of active site gating for E2s. For Ubc13, mutation of the key hinge residue A122 to glycine resulted in a faster rate of gate opening. This increase in the rate of opening, corresponds to a loss in the rate of aminolysis, as catalysis depends upon closing of the gate, to stabilize the zwitterionic charged transition state ${ }^{26}$. For E2-25K, the corresponding hinge residue is D127, and our combined MD and NMR relaxation data indicate that the gate is more flexible for the D127G mutant. Additionally, we estimate that the barrier to gate opening is decreased by $\sim 2 \mathrm{kcal} / \mathrm{mol}$ in comparison to wildtype, either by counting transitions during MD simulations, or through steered MD. Using our previously developed kinetic scheme for E2-catalyzed aminolysis ${ }^{26}$, this $2 \mathrm{kcal} / \mathrm{mol}$ decrease in barrier height for gate opening translates into an $\sim 7$-fold decrease in the rate of aminolysis for D127G at a lysine concentration of $30 \mathrm{mM}$, a value close to the experimentally determined 4-fold decrease. Interestingly, the D127G mutant is completely impaired with respect to catalysis of synthesis of $\mathrm{K} 48-\mathrm{Ub}_{2}$ chains. This result indicates a role for D127 in substrate binding and positioning, in addition to gating. Thus, as in the case of $\mathrm{Ubc}^{2} 3^{26}$, rates of opening and closing for the E2-25K active site gate appear to be precisely balanced, with an increase in the rate of gate opening resulting in loss of catalytic activity for aminolysis. However, there are some important differences in the gating mechanism of Ubc13 compared to E2-25K. Leucine at position Y within the gate of Ubc13 has only 
been observed to pack against the active site cysteine and adopt the closed conformation in the presence of the accessory protein Mms2 with Ub conjugated to the active site and bound to the underside of Ubc1 $3^{34}$, whereas E2-25K generally adopts the catalytically active, closed conformation. Thus, the fourfold larger second order rate constant for lysine aminolysis by E2-25K in comparison to $\mathrm{Ubc}_{13}{ }^{26}$, may be a result of E2-25K more effectively adopting the catalytically active closed conformation. It is instructive to compare our results for gating in E2-25K to those of other E2s. Cdc34, Ubc7, and Ube2g2 possess an acidic insertion within loop 7 (L7); a loop that is adjacent to the active site gate (loop 8, or L8). MD simulations have identified open and closed L7 conformations that affect the solvent accessibility of the active site $\mathrm{Cys}^{48,49}$. Interactions were observed between L7 and L8, and their dynamics were correlated. Phosphorylation of a serine on the central helix promotes an open conformation of L7 as a result of electrostatic repulsion and is thought to facilitate charging of E2 by E1. The acidic loop may also stabilize E2-Ub interactions. Residue 120 within L8 in human Ube2A, and its yeast homolog Rad6, is generally an acidic residue or a serine which can be phosphorylated. MD simulations indicate that phosphoryation of $S 120$ in Ube2a increases the solvent accessibility of the active site Cys, and that negative charge and residue size at this position are important for coordinating the substrate lysine $\mathrm{e}^{50}$.

It is interesting that the barrier to gate opening for E2-25K is relatively large, even though the rate constants for aminolysis and K48-linked ubiquitination for wildtype E2-25K are at least $10^{6}$-fold greater than the uncatalyzed rate constants, as previously observed for $\mathrm{Ubc} 3^{17}$. This result points to the important dual role of E3 ligases, not only do they provide target specificity for E2-catalyzed ubiquitination, they also provide a relatively small, but biologically crucial increase in the basic E2 rate of catalysis ${ }^{28}$, to ensure accurate target ubiquitination. Structural studies indicate that the RING-type E3 ligases stabilize the close packing of the donor, or thioester-linked Ub on the underside of the $\mathrm{E} 2$ in a closed conformation to enhance catalysis ${ }^{29,30}$. However, the relationship between E3-mediated ubiquitination to E2 active site gating and facilitating the closed E2 $\sim \mathrm{Ub}$ conformation is currently not well understood. In this regard, MD simulations for the SUMO-specific E2 Ubc9 in complex with SUMO (a Ub-like modifier), the E3 RanBP2, and substrate RanGAP1, indicate that the overall motions of Ubc9, including those of the substrate binding site, and L8, are reduced upon SUMO and E3 binding, with the dynamics around the active site becoming more correlated ${ }^{51,52}$. Interestingly, combined NMR and MD studies of the activation of Ube2g2 by two domains from the E3 gp78, the helix from the G2BR domain and the RING domain, have demonstrated that the E2 becomes more rigid upon binding G2BR and L7 with an acidic insertion becomes more closed. However, on subsequent binding of the RING domain from gp78, the extended L7 acidic loop equilibrates between being partially open and closed, which is believed to enable or facilitate subsequent substrate lysine $\operatorname{attack}^{53}$. Our previous study of the E2 enzyme Ubc13 $3^{17,26}$, and E2-25K in the present work, both in the absence of an E3 Ub ligase, indicate that opening and closing rates are precisely balanced. It will be of interest to determine if E3 ligases promote a more open gate to facilitate entry of the C-terminus of Ub into the E2 active site, or if the mechanism involves only stabilization of the close packing of Ub onto the underside of the E2.

\section{Methods}

Cloning, protein expression, and purification of E2-25K and active site gate mutants. Human E2-25K was cloned into the pHis-parallel1 vector. Site-directed mutagenesis was performed using a QuikChange mutagenesis kit to obtain the active site gate mutants D127G, Q126G, Q126L, Q126V, Q126A and Q126I. Expression of unlabeled protein was initiated by transforming $50 \mu \mathrm{L}$ of Epicurian coli BL21(DE3)-RIPL electro-competent cells with $100 \mathrm{ng}$ of plasmid DNA, followed by overnight growth on agar plates. A single colony was used to inoculate $50 \mathrm{~mL}$ of LB starter culture containing ampicillin, and grown overnight at $37^{\circ} \mathrm{C}$. Subsequently, $5 \mathrm{ml}$ of overnight starter culture were used to inoculate $500 \mathrm{~mL}$ of LB containing ampicillin and chlormaphenicol. Protein expression was induced through addition of $0.4 \mathrm{mM} 1$-thio- $\beta$-D-galactopyranoside upon growth of the cell culture to an $A_{600}$ of $\sim 0.6$, and incubated at $25^{\circ} \mathrm{C}$ overnight. For the production of [U- ${ }^{15} \mathrm{~N}$ ] and $\left[\mathrm{U}_{-}{ }^{13} \mathrm{C},{ }^{15} \mathrm{~N}\right] \mathrm{E} 2-25 \mathrm{~K}$ for NMR studies, M9 minimal medium containing $\left({ }^{15} \mathrm{NH}_{4}\right)_{2} \mathrm{SO}_{4}$ and ${ }^{13} \mathrm{C}$-glucose as the main nitrogen and carbon sources, respectively, was employed as described previously for the protein RAP8 $80^{54,55}$. Following protein overexpression, cells were harvested by centrifugation, suspended in $100 \mathrm{~mL}$ of lysis buffer $\left(500 \mathrm{mM} \mathrm{NaCl}, 20 \mathrm{mM}\right.$ imidazole, $1 \mathrm{mM}$ DTT, $100 \mu \mathrm{g} / \mathrm{ml}$ DNase I, $10 \mathrm{mM} \mathrm{MgSO}_{4}, 0.5 \%$ protease inhibitor cocktail and lysozyme), and lysed using sonication. The cell lysate was subjected to centrifugation, the supernatant was passed through a $0.22 \mu \mathrm{m}$ filter, and loaded onto a His-prep FF16/10 column equilibrated with binding buffer ( $20 \mathrm{mM}$ imidazole, $20 \mathrm{mM}$ sodium phosphate, $500 \mathrm{mM} \mathrm{NaCl}, 1 \mathrm{mM}$ DTT, pH 7.4). Following binding and washing with seven column volumes of binding buffer, E2-25K was eluted using a gradient of 20-500 mM imidazole achieved by increasing the proportion of elution buffer $(500 \mathrm{mM}$ imidazole, $20 \mathrm{mM}$ sodium phosphate, $500 \mathrm{mM}$ $\mathrm{NaCl}, 1 \mathrm{mM}$ DTT, pH 7.4) through the column. Fractions containing protein were collected and pooled, and the His affinity tag was cleaved using $150 \mu \mathrm{l}$ of $210 \mu \mathrm{M}$ TEV protease. The protein solution was dialyzed against PBS buffer (20 mM sodium phosphate, $150 \mathrm{mM} \mathrm{NaCl}$ and $1 \mathrm{mM}$ DTT, $\mathrm{pH} 7.4)$ using a $3.5 \mathrm{kDa}$ dialysis membrane at $20^{\circ} \mathrm{C}$. Dialyzed protein was passed through a $0.22 \mu \mathrm{m}$ filter and the affinity tag was separated from the solution by passing the sample over a His-prep FF 16/10 column. Purified protein was concentrated and passed through HiLoad 26/60 Superdex 75 column equilibrated with PBS buffer. The major fraction containing E2-25K was collected, concentrated to $500 \mu \mathrm{M}$, and stored at $-80^{\circ} \mathrm{C}$ in $10-500 \mu \mathrm{L}$ aliquots.

NMR spectroscopy. NMR samples were prepared by adjusting the concentrations of wildtype, Q126L, and D127G E2-25K stored at $-80{ }^{\circ} \mathrm{C}$ to $\sim 600-700 \mu \mathrm{M}$. NMR samples consisted of $\sim 300 \mu \mathrm{l}$ of $\left[\mathrm{U}-{ }^{15} \mathrm{~N}\right]$ or $\left[\mathrm{U}-{ }^{13} \mathrm{C},{ }^{15} \mathrm{~N}\right.$ ] protein in PBS buffer (20 mM sodium phosphate, $150 \mathrm{mM} \mathrm{NaCl}$ and $10 \mathrm{mM} \mathrm{DTT}$ at pH 7.4) containing $20 \mu \mathrm{l}$ of 99.9\% $\mathrm{D}_{2} \mathrm{O}$ and $1 \mathrm{mM}$ DSS. NMR experiments were recorded using a Varian Unity INOVA $600 \mathrm{MHz}$ spectrometer. Typically, NMR samples were buffer exchanged every three days with PBS buffer containing $10 \mathrm{mM}$ freshly dissolved DTT. Chemical shift assignments for E2-25K were accomplished using previous data ${ }^{36}$, and assignments deposited in the $\mathrm{BMRB}^{37}$. Assignment ambiguities were resolved through analysis of 3D HNCA, $\mathrm{HN}(\mathrm{CO}) \mathrm{CA}$, 
HNCACB, and CBCA(CO)NH experiments ${ }^{56-60}$. Assignment of the E2-25K mutants D127G and Q126L were accomplished using a combination of chemical shift data from the BMRB and the HNCA/HN(CO)CA experiments. Main chain amide ${ }^{15} \mathrm{~N}-R_{1}, R_{2}$, and ${ }^{1} \mathrm{H}-{ }^{15} \mathrm{~N}$ NOE relaxation experiments were recorded as described previously $^{61}$. The overall correlation times for molecular tumbling, or $\tau_{c}$ values, for E2-25K and the D127G/Q126L mutants were determined as previously described ${ }^{62,63}$. The impact of the D127G/Q126L mutations on the structure of E2-25K was assessed by determining main chain ${ }^{15} \mathrm{~N}$ and ${ }^{1} \mathrm{H}^{\mathrm{N}}$ chemical shift differences $(\Delta \delta)$ in $2 \mathrm{D}$ ${ }^{15} \mathrm{~N}-\mathrm{HSQC}$ NMR spectra according to:

$$
\Delta \delta=\sqrt{\left(\Delta \delta^{1} \mathrm{H}^{\mathrm{N}}\right)^{2}+\left(\Delta \delta^{15} \mathrm{~N} / 5\right)^{2}}
$$

where $\Delta \delta^{1} \mathrm{H}^{\mathrm{N}}$ and $\Delta \delta^{15} \mathrm{~N}$ are chemical shift differences of ${ }^{1} \mathrm{H}^{\mathrm{N}}$ and ${ }^{15} \mathrm{~N}$ dimension in $\mathrm{ppm}{ }^{64}$. NMR spectra were processed using the NMRpipe program ${ }^{65}$, and chemical shift assignments were accomplished using CARA ${ }^{66}$. Analyses of relaxation and chemical shift perturbation experiments were done using CCPNMR ${ }^{67}$. Secondary structures for wildtype, Q126L, D127G E2-25K were calculated from chemical shifts using the CSI 3.0 program $^{68}$.

NMR monitored titration and lineshape analysis for the E2-25K-Ub interaction. A stock solution of $\sim 800 \mu \mathrm{M}\left[\mathrm{U}_{-}{ }^{15} \mathrm{~N}\right]-\mathrm{E} 2-25 \mathrm{~K}$ was prepared in PBS buffer as described above. Lyophilized human ubiquitin was purchased from BostonBiochem and dissolved in identical PBS buffer as E2-25K to yield a stock concentration of $\sim 2 \mathrm{mM}$. The concentrations of both proteins were calculated using the bicinchoninic acid assay ${ }^{69}$, and verified with amino acid analyses. Six separate E2-25K samples were prepared from the same stock solution for the titration, having increasing concentrations of ubiquitin of $0,200,400,560,790$, and $1600 \mu \mathrm{M}$. The corresponding concentrations of E2-25K were 417, 420, 421, 424, 424, and $428 \mu \mathrm{M}$. All $2 \mathrm{D}{ }^{15} \mathrm{~N}$-HSQC NMR experiments were recorded using 32 scans and 128 increments in the ${ }^{15} \mathrm{~N}$ dimension. The combined change in ${ }^{1} \mathrm{H}^{\mathrm{N}}$ and ${ }^{15} \mathrm{~N}$ chemical shifts $(\Delta \delta)$, for each residue upon binding of ubiquitin to E2-25K were calculated according to Eq. $1 . \Delta \delta$ values were fit to a 1:1 binding isotherm to determine the dissociation constant $\left(K_{D}\right)$ as described previously ${ }^{70}$. Lineshape analysis was conducted to determine the kinetics of binding $\left(k_{o n}\right.$ and $k_{\text {off }}$ rates). Distinctly separated peaks in the ${ }^{15} \mathrm{~N}$ dimension, from the various spectra acquired for the titration, were fit using the Bloch-McConnell equations for two site exchange, as previously described ${ }^{71}$.

E1 conjugation assays. E2-25K samples stored at $-80^{\circ} \mathrm{C}$ were thawed, freshly reduced using $10 \mathrm{mM}$ DTT, and kept at $20^{\circ} \mathrm{C}$ overnight. The protein solution was dialyzed against $50 \mathrm{mM}$ HEPES buffer containing $150 \mathrm{mM}$ $\mathrm{NaCl}$ at $\mathrm{pH}$ 8.0. The concentration of E2-25K stock solution was adjusted to $260 \mu \mathrm{M}$ for all enzymatic assays. The ability of E1 to conjugate ubiquitin to E2-25K was quantified using reaction mixtures containing $35 \mathrm{mM}$ BTP (BIS-TRIS propane), $330 \mathrm{nM} \mathrm{E1,} 3 \mathrm{mM} \mathrm{MgCl}_{2}, 8 \mu \mathrm{M}$ E2-25K or D127G and Q126L mutants, $8 \mu \mathrm{M}$ ubiquitin labeled at the N-terminus with AlexaFluor488, and $3 \mathrm{mM}$ ATP. The reaction was allowed to proceed for 90 minutes, with samples collected at various time points, whose spacing was determined depending on the rate of conjugation. E2-25K Ub thioester conjugate was separated using SDS-PAGE, and quantitative analysis was accomplished using a Typhoon 9400 imager to detect AlexaFluor 488 fluorescence at $517 \mathrm{~nm}$. The observed rate of thioester buildup $\left(k_{o b s}\right)$ was determined using a two-parameter exponential fit. The apparent rate constant $\left(k_{a p p}\right)$, was determined by dividing $k_{o b s}$ by the $\mathrm{E} 1$ concentration to facilitate rate comparisons for the different mutants.

Hydrolysis Assays. The reaction mixtures for hydrolysis assays were similar to E1 conjugation assays. Hydrolysis was initiated by inhibiting the E1 catalyzed E2-25K Ub thioester conjugation reaction with $50 \mathrm{mM}$ PYR-41, an E1-specific inhibitor, and waiting 30 minutes to achieve complete inhibition ${ }^{72}$. Samples were collected at 20 min intervals, with quantification of E2-25K Ub thioester hydrolysis achieved using SDS-PAGE with imaging of AlexaFluor- 488 fluorescence as described above. The rates of thioester loss and concomitant build-up of $\mathrm{Ub}$ as a result of hydrolysis were calculated through fits to a two-parameter exponential decay for a first order reaction.

Aminolysis assays. Reaction mixtures for aminolysis were similar to those for E1 conjugation, as described above. Following conjugation of fluorescent Ub to E2-25K by E1, the reaction was inhibited by PYR-41, and various concentrations of lysine, ranging from 10 to $75 \mathrm{mM}$, were added to the reaction mixture. Samples were collected at regular time intervals, and SDS-PAGE was used to separate E2 Ub and Ub. Fluorescence intensities for $\mathrm{E} 2 \sim \mathrm{Ub}$ and $\mathrm{Ub}$ were fit to two parameter exponential decay and growth, to determine the rates of thioester loss and Ub build up, respectively. As previously described, the observed aminolysis rates were quadratic with respect to increasing lysine concentration, indicating that the rate law is given by ${ }^{26,73}$

$$
\frac{d[E 2 \sim U b]}{d t}=-k_{c a t, 1}[E 2 \sim U b]\left[l y s^{0}\right]-k_{c a t, 2}[E 2 \sim U b]\left[l y s^{0}\right]\left[l y s^{0}\right]
$$

where $l y s^{0}$ is concentration of lysine with neutral side chain at under the conditions of the reaction mixture. Plots of $k_{o b s} /\left[l y s^{0}\right]$ as a function of $\left[l y s^{0}\right]$ yield straight lines that are fit to obtain the rate constants $k_{c a t, 1}$ and $k_{c a t, 2}$.

Ubiquitination assays. Reaction mixtures were similar to those for E1 conjugation assays. Formation of thioester was inhibited after $\sim 30$ minutes by addition of PYR-41, and $\mathrm{K} 48-\mathrm{Ub}_{2}$ synthesis was initiated by addition of $100 \mu \mathrm{M}$ Ub lacking a fluorescent tag. $\mathrm{K} 48-\mathrm{Ub}_{2}$ synthesis was monitored by collecting aliquots at regular time intervals, separating the reaction mixtures using SDS-PAGE, followed by visualization and quantification of the gels by fluorescent imaging. To determine the catalytic rate constant for $\mathrm{K} 48-\mathrm{Ub}_{2}$ chain formation by $\mathrm{E} 2-25 \mathrm{~K}$ from these assays, the rate law corresponding to hydrolysis of thioester, ubiquitin binding to the UBA domain, and formation of $\mathrm{K} 48-\mathrm{Ub}_{2}$ was derived (Eq. 3): 


$$
\begin{aligned}
& \frac{d[E 2]}{d t}=k_{\mathrm{H}_{2} \mathrm{O}}[E 2 \sim U b]-k_{\text {on }}[E 2][U b] \\
& +k_{o f f}[E 2 \cdot U b]+k_{U b_{2}}[U b \cdot E 2 \sim U b] \\
& \frac{d[E 2 \sim U b]}{d t}=k_{\text {off }}[U b \cdot E 2 \sim U b]-k_{o n}[E 2 \sim U b][U b] \\
& -k_{\mathrm{H}_{2} \mathrm{O}}[E 2 \sim U b] \\
& \frac{d[U b \cdot E 2 \sim U b]}{d t}=-k_{\text {off }}[U b \cdot E 2 \sim U b]+k_{o n}[E 2 \sim U b][U b] \\
& -k_{U b_{2}}[U b \cdot E 2 \sim U b]-k_{H_{2} \mathrm{O}}[U b \cdot E 2 \sim U b] \\
& \frac{d[E 2 \cdot U b]}{d t}=-k_{o f f}[U b \cdot E 2]+k_{o n}[E 2][U b]+k_{H_{2} O}[U b \cdot E 2 \sim U b] \\
& \frac{d[U b]}{d t}=k_{o f f}[U b \cdot E 2 \sim U b]+k_{o f f}[U b \cdot E 2]-k_{o n}[E 2 \sim U b][U b] \\
& -k_{o n}[E 2][U b]+k_{\mathrm{H}_{2} \mathrm{O}}[E 2 \sim U b]+k_{\mathrm{H}_{2} \mathrm{O}}[U b \cdot E 2 \sim U b] \\
& \frac{d\left[U b_{2}\right]}{d t}=k_{U b_{2}}[U b \cdot E 2 \sim U b]
\end{aligned}
$$

where the kinetics of Ub binding to the UBA domain are given by the on and off-rates $\left(k_{o n}, k_{o f f}\right)$. Hydrolysis of the $\mathrm{E} 2 \sim \mathrm{Ub}$ thioester is given by the rate constant $k_{\mathrm{H}_{2} \mathrm{O}}$, and the catalytic rate constant for $\mathrm{K} 48-\mathrm{Ub}_{2}$ formation given by $k_{\mathrm{Ub} 2}$. The catalytic rate constant $k_{\mathrm{Ub} 2}$ was obtained from numerical integration of the coupled differential equations (eq. 3), followed by numerical optimization of $k_{\mathrm{Ub} 2}$ (Fig. 7b). The rate constants $k_{o n}$ and $k_{o f f}$, describing the reversible binding of "acceptor" Ub to UBA domain, determined using ${ }^{15} \mathrm{~N}$ NMR lineshape analysis, were used for the numerical optimization. The on and off rates of Ub binding to the UBA domain of E2-25K thioester were assumed to be similar to those for unconjugated E2-25K.

Molecular dynamics simulations for E2-25K and the Q126L/D127G active site gate mutants. A starting model for E2-25K was derived from the crystallographically determined structure (3K9O). All simulations were performed using the f99SBNMR force field within the AMBER 11 or 12 suite of biomolecular simulation programs ${ }^{74}$. Structural models for the mutants were generated from the crystal structure (3K9O) using the mutagenesis protocol within the Pymol program. Starting models were then solvated within an octahedral box with a minimum of $12 \AA$ between protein atoms and the box edges using the TIP3P water model. $\mathrm{Na}^{+}$ions were added to balance the net negative charge on E2-25K and ensure system neutrality. The steepest descent method was used for initial energy minimization followed by conjugate gradient minimization. Bonds involving hydrogen atoms were constrained using the SHAKE algorithm. The system temperature was managed using Langevin dynamics with a collision frequency of $1 \mathrm{ps}^{-1}$. Pairwise non-bonded and electrostatic interactions were calculated using a particle mesh Ewald approach and a distance cutoff of $8 \AA$. The system was heated from 0 to $298 \mathrm{~K}$ over $50 \mathrm{ps}$ with $0.1 \mathrm{kcal} / \mathrm{mol}$ restraints applied to all the solute atoms, followed by equilibration to 1 atm pressure for 50 ps. Typically two to five $\sim 400-800$ ns production dynamic runs were conducted for each of the D127G/Q126L mutants and wildtype E2-25K. The total simulation times for wildtype, D127G, and Q126L E2-25K were 3.56, 2.83 , and $1.62 \mu \mathrm{s}$, respectively.

Time dependent fluctuations of the active site gate were determined from the MD simulations by calculating the distance between the $\mathrm{C}_{\alpha}$ atoms of the active site C92 and Q126 (or Q126L) from the active site loop over the duration of the simulation. For distances exceeded $12 \AA$, the gate was considered to be in an open conformation, otherwise the gate was considered to be in a closed conformation. The rates of opening and closing were determined by dividing the number of open or closed transitions by the total time in the open or closed states, respectively. Simulations for E2-25K with covalently attached ubiquitin were initially set up as described previously ${ }^{26,75} \cdot \mathrm{C}_{\alpha}-\mathrm{C}_{\alpha}$ restraints and a restraint between E2-25K N83 HD22 and Ub G76 O were used during equilibration of the structure, after which the restraints were gradually relaxed over $4 \mathrm{~ns}$. Two to three runs of $\sim 400-800 \mathrm{~ns}$ for each of WT, Q126L, and D127G thioester-bound E2-25K were performed, for a total of $2.19,1.59$, and $2.34 \mu \mathrm{s}$, respectively.

Steered MD simulations were performed in AMBER to investigate the energetic barrier to the opening of the active site gate $^{76}$. WT, Q126L, and D127G E2-25K systems were generated and equilibrated as described above, then equilibrated for a further $100 \mathrm{ps}$ with a $10 \mathrm{kcal} / \mathrm{mol} / \AA^{2}$ restraint between the $\mathrm{Q} / \mathrm{L} 126 \mathrm{C}_{\alpha}$ in the gate and the center of protein, as defined by the average position of the $\mathrm{C}_{\alpha}$ atoms of E2-25K but not including the gate (residues 122-128). After equilibration, the restraint distance was increased at a speed of $0.3 \AA / \mathrm{ns}$, and the work performed on the system by pulling the restraint was monitored as a function of the restraint distance. A total of 150 runs for each protein were performed, and the barrier heights were calculated using the cumulant expansion method ${ }^{77}$.

\section{References}

1. Lim, J. \& Yue, Z. Neuronal aggregates: formation, clearance, and spreading. Dev Cell 32, 491-501, https://doi.org/10.1016/j. devcel.2015.02.002 (2015).

2. Ortega, Z. \& Lucas, J. J. Ubiquitin-proteasome system involvement in Huntington's disease. Front Mol Neurosci 7, 77, https://doi. org/10.3389/fnmol.2014.00077 (2014).

3. Atkin, G. \& Paulson, H. Ubiquitin pathways in neurodegenerative disease. Front Mol Neurosci 7, 63, https://doi.org/10.3389/ fnmol.2014.00063 (2014). 
4. Pickart, C. M. \& Eddins, M. J. Ubiquitin: structures, functions, mechanisms. Biochim Biophys Acta 1695, 55-72, https://doi. org/10.1016/j.bbamcr.2004.09.019 (2004).

5. Deshaies, R. J. SCF and Cullin/Ring H2-based ubiquitin ligases. Annu Rev Cell Dev Biol 15, 435-467 (1999).

6. Haglund, K., Di Fiore, P. P. \& Dikic, I. Distinct monoubiquitin signals in receptor endocytosis. Trends Biochem Sci 28, 598-603 (2003).

7. Chen, Z. J., Parent, L. \& Maniatis, T. Site-specific phosphorylation of $I \kappa B \alpha$ by a novel ubiquitination-dependent protein kinase activity. Cell 84, 853-862 (1996).

8. Hochstrasser, M. Ubiquitin-dependent protein degradation. Annu Rev Genet 30, 405-439 (1996).

9. Hoege, C., Pfander, B., Moldovan, G. L., Pyrowolakis, G. \& Jentsch, S. RAD6-dependent DNA repair is linked to modification of PCNA by ubiquitin and SUMO. Nature 419, 135-141 (2002).

10. Sobhian, B. et al. RAP80 targets BRCA1 to specific ubiquitin structures at DNA damage sites. Science 316, 1198-1202 (2007).

11. Lee, B. L., Singh, A., Mark Glover, J. N., Hendzel, M. J. \& Spyracopoulos, L. Molecular basis for K63-linked ubiquitination processes in double-strand DNA break repair: A focus on kinetics and dynamics. J Mol Biol 429, 3409-3429, https://doi.org/10.1016/j. jmb.2017.05.029 (2017).

12. Wang, C. et al. TAK1 is a ubiquitin-dependent kinase of MKK and IKK. Nature 412, 346-351 (2001).

13. Shaid, S., Brandts, C. H., Serve, H. \& Dikic, I. Ubiquitination and selective autophagy. Cell Death Differ 20, 21-30, https://doi. org/10.1038/cdd.2012.72 (2013).

14. Glickman, M. H. \& Ciechanover, A. The ubiquitin-proteasome proteolytic pathway: Destruction for the sake of construction. Physiol $\operatorname{Rev} 82,373-428$ (2002)

15. Nikkila, J. et al. Familial breast cancer screening reveals an alteration in the RAP80 UIM domain that impairs DNA damage response function. Oncogene 28, 1843-1852, https://doi.org/10.1038/onc.2009.33 (2009).

16. Pierce, N. W., Kleiger, G., Shan, S. O. \& Deshaies, R. J. Detection of sequential polyubiquitylation on a millisecond timescale. Nature 462, 615-619, https://doi.org/10.1038/nature08595 (2009).

17. Markin, C. J. et al. Catalytic proficiency of ubiquitin conjugation enzymes:. Balancing $p K_{\mathrm{a}}$ suppression, entropy, and electrostatics. J Am Chem Soc 132, 17775-17786 (2010).

18. Varshavsky, A. The ubiquitin system, an immense realm. Annu Rev Biochem 81, 167-176, https://doi.org/10.1146/annurevbiochem-051910-094049 (2012).

19. Komander, D. The emerging complexity of protein ubiquitination. Biochem Soc Trans 37, 937-953, https://doi.org/10.1042/ BST0370937 (2009).

20. Swatek, K. N. \& Komander, D. Ubiquitin modifications. Cell Res 26, 399-422, https://doi.org/10.1038/cr.2016.39 (2016).

21. Song, S. et al. Essential role of E2-25K/Hip-2 in mediating amyloid-beta neurotoxicity. Mol Cell 12, 553-563 (2003).

22. Kalchman, M. A. et al. Huntingtin is ubiquitinated and interacts with a specific ubiquitin-conjugating enzyme. J Biol Chem 271, 19385-19394 (1996).

23. de Pril, R., Fischer, D. F., Roos, R. A. \& van Leeuwen, F. W. Ubiquitin-conjugating enzyme E2-25K increases aggregate formation and cell death in polyglutamine diseases. Mol Cell Neurosci 34, 10-19, https://doi.org/10.1016/j.mcn.2006.09.006 (2007).

24. Martin, D. D., Ladha, S., Ehrnhoefer, D. E. \& Hayden, M. R. Autophagy in Huntington disease and huntingtin in autophagy. Trends Neurosci 38, 26-35, https://doi.org/10.1016/j.tins.2014.09.003 (2015).

25. Bhat, K. P., Yan, S., Wang, C. E., Li, S. \& Li, X. J. Differential ubiquitination and degradation of huntingtin fragments modulated by ubiquitin-protein ligase E3A. Proc Natl Acad Sci USA 111, 5706-5711, https://doi.org/10.1073/pnas.1402215111 (2014).

26. Rout, M. K. et al. Stochastic gate dynamics regulate the catalytic activity of ubiquitination enzymes. J Am Chem Soc 136, 17446-17458, https://doi.org/10.1021/ja505440b (2014).

27. Ye, Y. \& Rape, M. Building ubiquitin chains: E2 enzymes at work. Nat Rev Mol Cell Biol 10, 755-764, https://doi.org/10.1038/ nrm2780 (2009).

28. Ozkan, E., Yu, H. \& Deisenhofer, J. Mechanistic insight into the allosteric activation of a ubiquitin-conjugating enzyme by RINGtype ubiquitin ligases. Proc Natl Acad Sci USA 102, 18890-18895, https://doi.org/10.1073/pnas.0509418102 (2005).

29. Pruneda, J. N. et al. Structure of an E3:E2 Ub complex reveals an allosteric mechanism shared among RING/U-box ligases. Mol Cell 47, 933-942 (2012)

30. Plechanovova, A., Jaffray, E. G., Tatham, M. H., Naismith, J. H. \& Hay, R. T. Structure of a RING E3 ligase and ubiquitin-loaded E2 primed for catalysis. Nature 489, 115-120, https://doi.org/10.1038/nature11376 (2012).

31. Hodge, C. D. et al. Covalent inhibition of Ubc13 affects ubiquitin signaling and reveals active site elements important for targeting. ACS Chem Biol 10, 1718-1728, https://doi.org/10.1021/acschembio.5b00222 (2015).

32. Moraes, T. F. et al. Crystal structure of the human ubiquitin conjugating enzyme complex, hMms2-hUbc13. Nat Struct Biol 8 , 669-673 (2001)

33. Eddins, M. J., Carlile, C. M., Gomez, K. M., Pickart, C. M. \& Wolberger, C. Mms2-Ubc13 covalently bound to ubiquitin reveals the structural basis of linkage-specific polyubiquitin chain formation. Nat Struct Mol Biol 13, 915-920 (2006).

34. Branigan, E., Plechanovova, A., Jaffray, E. G., Naismith, J. H. \& Hay, R. T. Structural basis for the RING-catalyzed synthesis of K63linked ubiquitin chains. Nat Struct Mol Biol 22, 597-602, https://doi.org/10.1038/nsmb.3052 (2015).

35. Warshel, A., Aqvist, J. \& Creighton, S. Enzymes work by solvation substitution rather than by desolvation. Proc Natl Acad Sci USA 86, 5820-5824 (1989).

36. Ko, S. et al. Structural basis of E2-25K/UBB +1 interaction leading to proteasome inhibition and neurotoxicity. J Biol Chem 285, 36070-36080, https://doi.org/10.1074/jbc.M110.145219 (2010).

37. Wilson, R. C., Edmondson, S. P., Flatt, J. W., Helms, K. \& Twigg, P. D. The E2-25K ubiquitin-associated (UBA) domain aids in polyubiquitin chain synthesis and linkage specificity. Biochem Biophys Res Commun 405, 662-666, https://doi.org/10.1016/j. bbrc.2011.01.089 (2011).

38. Merkley, N. \& Shaw, G. S. Solution structure of the flexible class II ubiquitin-conjugating enzyme Ubc1 provides insights for polyubiquitin chain assembly. J Biol Chem 279, 47139-47147, https://doi.org/10.1074/jbc.M409576200 (2004).

39. Rodrigo-Brenni, M. C., Foster, S. A. \& Morgan, D. O. Catalysis of lysine 48-specific ubiquitin chain assembly by residues in E2 and ubiquitin. Mol Cell 39, 548-559, https://doi.org/10.1016/j.molcel.2010.07.027 (2010).

40. Pickart, C. M. \& Rose, I. A. Functional heterogeneity of ubiquitin carrier proteins. J Biol Chem 260, 1573-1581 (1985).

41. Chen, Z. \& Pickart, C. M. A 25-kilodalton ubiquitin carrier protein (E2) catalyzes multi-ubiquitin chain synthesis via lysine 48 of ubiquitin. J Biol Chem 265, 21835-21842 (1990).

42. Cook, B. W. \& Shaw, G. S. Architecture of the catalytic HPN motif is conserved in all E2 conjugating enzymes. Biochem J 445, 167-174, https://doi.org/10.1042/BJ20120504 (2012).

43. Showalter, S. A. \& Bruschweiler, R. Validation of molecular dynamics simulations of biomolecules using NMR spin relaxation as benchmarks: Application to the AMBER99SB force field. J Chem Theory Comput 3, 961-975, https://doi.org/10.1021/Ct7000045 (2007).

44. Stewart, M. D., Ritterhoff, T., Klevit, R. E. \& Brzovic, P. S. E2 enzymes: more than just middle men. Cell Res 26, 423-440, https://doi. org/10.1038/cr.2016.35 (2016)

45. Reverter, D. \& Lima, C. D. Insights into E3 ligase activity revealed by a SUMO-RanGAP1-Ubc9-Nup358 complex. Nature 435, 687-692 (2005)

46. Wu, P. Y. et al. A conserved catalytic residue in the ubiquitin-conjugating enzyme family. EMBO J 22, 5241-5250 (2003). 
47. Berndsen, C. E., Wiener, R., Yu, I. W., Ringel, A. E. \& Wolberger, C. A conserved asparagine has a structural role in ubiquitinconjugating enzymes. Nat Chem Biol 9, 154-156, https://doi.org/10.1038/nchembio.1159 (2013).

48. Papaleo, E. et al. Loop 7 of E2 enzymes: an ancestral conserved functional motif involved in the E2-mediated steps of the ubiquitination cascade. Plos One 7, e40786, https://doi.org/10.1371/journal.pone.0040786 (2012).

49. Papaleo, E. et al. An acidic loop and cognate phosphorylation sites define a molecular switch that modulates ubiquitin charging activity in Cdc34-like enzymes. PLoS Comput Biol 7, e1002056, https://doi.org/10.1371/journal.pcbi.1002056 (2011).

50. Valimberti, I., Tiberti, M., Lambrughi, M., Sarcevic, B. \& Papaleo, E. E2 superfamily of ubiquitin-conjugating enzymes: constitutively active or activated through phosphorylation in the catalytic cleft. Sci Rep 5, 14849, https://doi.org/10.1038/srep14849 (2015).

51. Karaca, E., Tozluoglu, M., Nussinov, R. \& Haliloglu, T. Alternative allosteric mechanisms can regulate the substrate and E2 in SUMO conjugation. J Mol Biol 406, 620-630, https://doi.org/10.1016/j.jmb.2010.12.044 (2011).

52. Tozluoglu, M., Karaca, E., Nussinov, R. \& Haliloglu, T. A mechanistic view of the role of E3 in sumoylation. Plos Comput Biol 6, https://doi.org/10.1371/journal.pcbi.1000913 (2010).

53. Chakrabarti, K. S., Li, J., Das, R. \& Byrd, R. A. Conformational dynamics and allostery in E2:E3 interactions drive ubiquitination: gp78 and Ube2g2. Structure 25(794-805), e795, https://doi.org/10.1016/j.str.2017.03.016 (2017).

54. Marley, J., Lu, M. \& Bracken, C. A method for efficient isotopic labeling of recombinant proteins. J Biomol NMR 20, 71-75 (2001).

55. Anamika, M. C. J., Rout, M. K. \& Spyracopoulos, L. Molecular basis for impaired DNA damage response function associated with the RAP80 $\Delta$ E81 defect. J Biol Chem 289, 12852-12862, https://doi.org/10.1074/jbc.M113.538280 (2014).

56. Muhandiram, D. R. \& Kay, L. E. Gradient-enhanced triple-resonance three-dimensional NMR experiments with improved sensitivity. J Magn Reson B103, 203-216 (1994).

57. Kay, L. E., Ikura, M., Tschudin, R. \& Bax, A. 3-Dimensional triple-resonance NMR-spectroscopy of isotopically enriched proteins. J Magn Reson 89, 496-514 (1990)

58. Wittekind, M. \& Mueller, L. HNCACB, a high-sensitivity 3D NMR experiment to correlate amide proton and nitrogen resonances with the alpha and beta-carbon resonances in proteins. J Magn Reson B101, 201-205 (1993).

59. Grzesiek, S. \& Bax, A. Correlating backbone amide and side chain resonances in larger proteins by multiple relayed triple resonance NMR. J Am Chem Soc 114, 6291-6293 (1992).

60. Bax, A. \& Ikura, M. An efficient 3D NMR technique for correlating the proton and ${ }^{15} \mathrm{~N}$ backbone amide resonances with the alphacarbon of the preceding residue in uniformly ${ }^{15} \mathrm{~N} /{ }^{13} \mathrm{C}$ enriched proteins. J Biomol NMR 1, 99-104 (1991).

61. Farrow, N. A. et al. Backbone dynamics of a free and a phosphopeptide-complexed Src homology-2 domain studied by ${ }^{15} \mathrm{~N}$ NMR relaxation. Biochemistry 33, 5984-6003 (1994).

62. Kay, L. E., Torchia, D. A. \& Bax, A. Backbone dynamics of proteins as studied by ${ }^{15} \mathrm{~N}$ inverse detected heteronuclear NMR spectroscopy: Application to staphylococcal nuclease. Biochemistry 28, 8972-8979 (1989).

63. Spyracopoulos, L. A suite of Mathematica notebooks for the analysis of protein main chain ${ }^{15} \mathrm{~N}$ NMR relaxation data. J Biomol NMR 36, 215-224 (2006).

64. Garrett, D. S. et al. Solution structure of the $30 \mathrm{kDa} \mathrm{N}$-terminal domain of enzyme I of the escherichia coli phsphoenolpyruvate:sugar phosphotransferase system by multidimensional NMR. Biochemistry 36, 2517-2530 (1997).

65. Delaglio, F. et al. NMRPipe: A multidimensional spectral processing system based on UNIX pipes. J Biomol NMR 6, 277-293 (1995).

66. Keller, R. L. J. CARA: The computer-aided resonance assignment tutorial. Cantina Verlag, Zürich, Switzerland. URL cara.nmr.ch/ (2004).

67. Vranken, W. F. et al. The CCPN data model for NMR spectroscopy: Development of a software pipeline. Proteins 59, 687-696, https://doi.org/10.1002/prot.20449 (2005).

68. Hafsa, N. E., Arndt, D. \& Wishart, D. S. CSI 3.0: a web server for identifying secondary and super-secondary structure in proteins using NMR chemical shifts. Nucleic Acids Res 43, W370-377, https://doi.org/10.1093/nar/gkv494 (2015).

69. Smith, P. K. et al. Measurement of protein using bicinchoninic acid. Anal Biochem 150, 76-85 (1985).

70. Markin, C. J. \& Spyracopoulos, L. Increased precision for analysis of protein-ligand dissociation constants determined from chemical shift titrations. J Biomol NMR 53, 125-138 (2012).

71. Markin, C. J. \& Spyracopoulos, L. Accuracy and precision of protein-ligand interaction kinetics determined from chemical shift titrations. J Biomol NMR 54, 355-376 (2012).

72. Yang, Y. et al. Inhibitors of ubiquitin-activating enzyme (E1), a new class of potential cancer therapeutics. Cancer Res 67, 9472-9481, https://doi.org/10.1158/0008-5472.CAN-07-0568 (2007).

73. Connors, K. A. \& Bender, M. L. Kinetics of alkaline hydrolysis and N-butylaminolysis of ethyl p-nitrobenzoate and ethyl $p$ nitrothiolbenzoate. J Org Chem 26, 2498-2504 (1961).

74. Case, D. A. et al. AMBER 11: A suite of biomolecular simulation programs. University of California, San Fransisco, USA. URL ambermd.org/ (2010).

75. Hau, D. D. et al. Structure and interactions of the ubiquitin-conjugating enzyme variant human Uevla: Implications for enzymatic synthesis of polyubiquitin chains. Biochemistry 45, 9866-9877 (2006).

76. Crespo, A., Marti, M. A., Estrin, D. A. \& Roitberg, A. E. Multiple-steering QM-MM calculation of the free energy profile in chorismate mutase. J Am Chem Soc 127, 6940-6941 (2005).

77. Park, S., Khalili-Araghi, F., Tajkhorshid, E. \& Schulten, K. Free energy calculation from steered molecular dynamics simulations using Jarzynski's equality. J Chem Phys 119, 3559-3566, https://doi.org/10.1063/1.1590311 (2003).

\section{Acknowledgements}

This work was supported through a grant from the Canadian Institutes of Health Research (CIHR) to L.S. (MOP 110964), and Natural Sciences and Engineering Research Council (NSERC) Discovery grants to L.S. (201605778) and W.X. (2014-04580).

\section{Author Contributions}

L.S., M.K.R., and B.L.L. conceived the project and wrote the main manuscript. A.L. and W.X. performed cloning and mutagenesis. All authors reviewed and commented on the manuscript at all stages.

\section{Additional Information}

Supplementary information accompanies this paper at https://doi.org/10.1038/s41598-018-25476-8.

Competing Interests: The authors declare no competing interests.

Publisher's note: Springer Nature remains neutral with regard to jurisdictional claims in published maps and institutional affiliations. 
(i) Open Access This article is licensed under a Creative Commons Attribution 4.0 International License, which permits use, sharing, adaptation, distribution and reproduction in any medium or format, as long as you give appropriate credit to the original author(s) and the source, provide a link to the Creative Commons license, and indicate if changes were made. The images or other third party material in this article are included in the article's Creative Commons license, unless indicated otherwise in a credit line to the material. If material is not included in the article's Creative Commons license and your intended use is not permitted by statutory regulation or exceeds the permitted use, you will need to obtain permission directly from the copyright holder. To view a copy of this license, visit http://creativecommons.org/licenses/by/4.0/.

(C) The Author(s) 2018 